\title{
Design variability verification in Software Product Lines
}

\author{
GANESH KHANDU NARWANE ${ }^{1, *}$, JEAN-VIVIEN MILLO $^{2}$, \\ SHANKARA NARAYANAN KRISHNA ${ }^{3}$ and $\mathrm{S} \mathrm{RAMESH}^{2}$ \\ ${ }^{1}$ Homi Bhabha National Institute, Mumbai 400094, India \\ ${ }^{2}$ Global General Motors R\&D, TCI Bangalore, Bangalore, India \\ ${ }^{3}$ Department of Computer Science and Engineering, Indian Institute of Technology Bombay, Mumbai, India \\ e-mail: ganeshk@cse.iitb.ac.in
}

MS received 15 May 2017; revised 30 August 2017; accepted 7 July 2018; published online 2 January 2019

\begin{abstract}
This paper proposes a novel notion called variability verification applicable to Software Product Lines (SPL). Variability is central to SPL and we have observed that variability is expressed differently at different levels of abstraction in the development flow of SPL. A natural problem in this context is the conformance of variability information expressed at different levels. Design variability verification, in particular, checks whether the variability expressed at the design level conforms to that at the requirement level. Unlike many existing approaches to SPL modelling, our work does not assume a single global view of variation points, even within the same level of abstraction. In our view, an SPL is a concurrent composition of features, where each feature exhibits independent variability. This enables incremental addition of variability. The procedure is compositional in the sense that the verification of an entire SPL consisting of multiple features is reduced to the verification of the individual features. Feature level verification essentially involves standard model checking while, in the second step, a Quantified Boolean Formula (QBF) is synthesized and solved. The method has been implemented and demonstrated in a tool SPL Engine for Design Verification (SPLEnD) on a couple of fairly large case studies. SPLEnD uses SPIN tool for the feature level conformance, while the state-of-the-art QBF solver CirQit is used for the SPL level conformance. SPLEnD easily handles the evolution of SPL by addition of new features and modification of existing features. Experimental results with SPLEnD look very promising.
\end{abstract}

Keywords. Software Product Lines; variability verification; SPIN; QBF.

\section{Introduction}

Large industrial software are often developed as Software Product Lines (SPLs) with a common core set of features, which are developed once and reused across all the products of the family. The products in an SPL differ on a small set of features, which are specified using variation points.

A central aspect of SPLs is variability modelling and analysis; many early works on SPL modelling focused on this. One of the most prominent models for capturing variability is feature diagrams [1]. Feature diagrams (as well as other variability description formalisms) assume a global view of SPL as they start with a complete list of features and the variation points using a single vocabulary. All the subsequent SPL assets, like requirement documents, design models, source codes, test cases and documentations, share the same definition and vocabulary as presented by [2] and [3].

However, the assumption of a single homogeneous and transverse view of variability description across the entire

*For correspondence life cycle of software development seems to impose a waterfall model of development that is limited and inflexible. We give a few instances of the limitations.

First, SPL developers naturally tend to use different representations and vocabulary of variability at different stages of development: at the requirement level, a more abstract and intuitive description of variation points is used, while at the design level, the efficiency of implementation of variation points is of primary concern. For example, consider the case of an automotive SPL, where one variation point is the region of sales (e.g., Asia Pacific, Europe, North America, South America and Africa). At the requirement level, this variation point is expressed directly as an enumeration variable assuming one value for every region. However, at the design level, the variation point is expressed using two Boolean variables; by setting the values of the Boolean variables appropriately, the behaviour specific to one of the four regions is selected at the time of deployment.

Second, SPLs often evolve during their long lifetime as new features and variabilities are added, removed or combined during the evolution. A unique and transverse view of 
the variability requires maintaining the global consistency. A simple change such as combining two variation points into one or splitting one into many requires propagating this change in every asset impacted at every level of abstraction (from requirement document to test case) and there is not yet an automatic method to perform these changes automatically and consistently. Here one can cite a preliminary attempt in this direction by [4].

Third, the end user companies use off-the-shelf components (hardware or software) to implement their features. For example, the Adaptive Cruise Controller (ACC) feature of an automotive SPL can be implemented by either an offthe-shelf ACC component or a combination of off-the-shelf sub-components such as the tracking system (using radar), maximal authorized speed detection system (using GPS map information) and an in-house sub-component depending upon the company-specific philosophy. In both cases, there is a distinction (at least in the vocabulary) between the variability as expressed in the SPL where company-specific philosophy (and keyword) would be respected and the variability offered by the off-the-shelf component that is as generic as possible even though it might be domain specific. The management of this distinction in a single homogeneous and transverse view of the variability would impose a very rigid development methodology.

Fourth, the features are not Boolean anymore thanks to [5]. It is not enough to say that a product line of personal computers offers printing facilities. One would like to specify whether it is $2 D$ or $3 D$ printing, black and white or colour, A2, A3 or $A 4$. One can add sub-features in the feature diagram but such addition tends to overload the feature diagram and increase the number of cross-tree constraints. Instead, it is natural to consider that a feature has parameters.

In order to overcome this limitation, we propose an SPL modelling framework that does not start with a global view of variability. The framework allows different vocabularies of variability for different levels of abstraction: the requirement models can use one vocabulary for expressing variability while the design models can employ a different variability vocabulary. Further, the proposed framework is compositional as it allows addition of variabilities as new features are added. The constraints relating variabilities in different functions can be added along with them. Further, the framework supports both Boolean and non-Boolean variabilities with multiple attributes.

One important problem with the use of different variability descriptions at different levels of abstraction is to maintain the consistency across the levels. Consider the automotive SPL described earlier, where one variation point at the requirement level is the region of sale. The same variability is described at the design level as a pair of Boolean values. For consistency, we need to check, for example, that the behaviour of the product when the variation point assumes the value European market, at the requirement level, matches with that of the design with the variability expressed at the design level as a pair of Boolean values (true, false). In SPL based on a single view of variability, such a mapping is trivial and no special checks are required.

We refer to this problem generically as variability verification and when the consistency of variabilities is expressed at the design and requirement levels, we call this design variability verification. We present here a solution based on formal methods to this problem. This proposed solution is based upon a simple notion of consistency of variabilities at the design and requirement levels: for every variability at the design level (or loosely, for every product at the design level), there is a variability at the requirement level (again loosely, a product at the requirement level), such that the behaviour of the design corresponds to that of the requirement.

In this paper, we will limit framework and analyses to only the requirement and design level of abstraction. For the formalization, the proposed framework extends the standard finite state machine model, which we call Finite State Machines with Variability, in short, FSMv. The behaviour and variability of a feature at the requirement and design levels can be modelled using FSMv. We define a conformance relation between FSMvs to relate the requirement and design models. This relation is based upon the standard language containment of state machines.

One unique feature of FSMv is that it provides a compositional operator for composing the feature state machines. The variability information in a state machine is not derived from a single global variability description and hence incremental addition of features and variabilities is possible using this operator.

One challenge in the variability verification is the analysis complexity: the number of products is exponential in the number of variation points and hence product-centric analyses are not scalable. We propose a compositional approach in which every feature of the SPL is first analysed independently; the per-feature analysis results are then combined to get the analysis result for the whole SPL. The proposed verification approach exploits the compositional structure of the FSMv models to contain the analysis complexity.

The variability verification is to be contrasted with the many recent works on SPL behaviour verification in [6] and [7]. The latter works focus on property-based verification of SPLs, which involves checking whether a property holds for all products in an SPL or if not which products do not satisfy the property. In contrast, the variability verification is concerned with the correspondence of design level variability with that of requirements.

Figure 1 summarizes the proposed approach. It shows an SPL composed of features $f_{1}$ to $f_{n}$. Each feature has an FSMv model of its requirements (called FSMr) and an FSMv model derived from its design (called FSMd). The proposed analysis method checks whether the FSMd of 


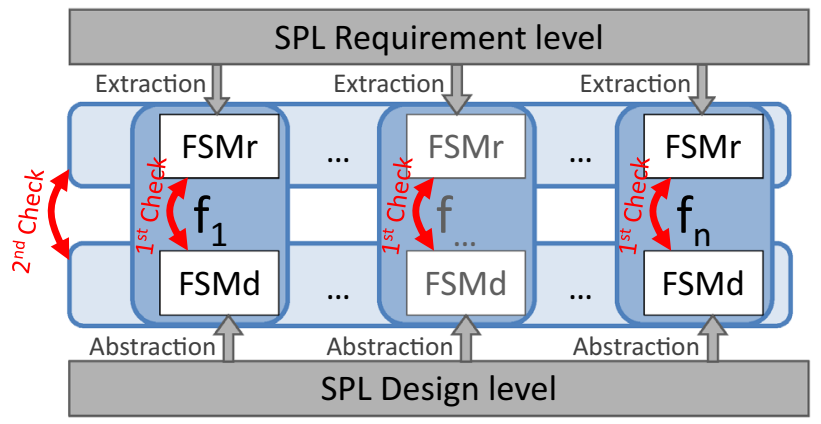

Figure 1. The proposed verification approach.

every feature conforms to its FSMr ( $1^{\text {st }}$ check). The output of this first step is a conformance relation $\Phi_{i}$ between each pair of $F S M r_{i}$ and $F S M d_{i}$. The obtained conformance relations $\Phi_{1}, \ldots, \Phi_{n}$ are then used to check whether the actual behaviour of the entire SPL conforms to the expected one ( $2^{\text {nd }}$ check). The $2^{\text {nd }}$ check is done by synthesizing a Quantified Boolean Formula (QBF) and answering its satisfiability. There is no need to build the entire behavioural model of the SPL in the second step. We have built a tool SPLEnD (SPL Engine for Design Verification) based upon this approach. This tool performs the first check using SPIN [8], while the well-known QBF SAT solver CirQit [9] is used for the second step. We have experimented with the tool using modest industrial size examples with very encouraging results.

Below is the Entry Control Product Line (ECPL), which is used to understand our work in upcoming sections.

\section{$1.1 E C P L$}

This is an SPL from the automotive domain. The ECPL comprises all the features involved in the management of the locks in a car. In this study, we focus on the following features.

- Power lock: this is the basic locking functionality that manages the locking/unlocking according to key button press and courtesy switch press.

- Last Door Closed lock: delays the locking of the doors until all the doors are closed. It is applicable when the lock command appends while a door is open.

- Door lock: automates the locking of doors when the vehicle starts.

- Door unlock: automates the unlocking of door(s) when the vehicle stops.

- Anti-lockout: this is intended to prevent the inadvertent lockout situations - the driver is out of the car with the key inside and all the doors locked.

- Post-crash unlock: unlocks all the doors in a post-crash situation.

- Theft security lock: secures the car with a second lock.

- Lift glass: manages the opening of the lift glass, when present.

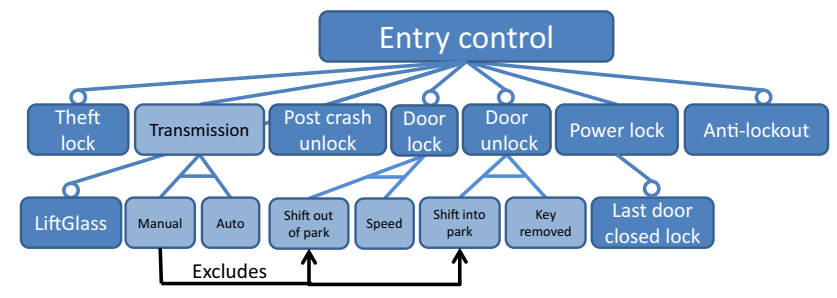

Figure 2. The feature diagram of the ECPL.

Figure 2 presents the feature diagram of the ECPL [2]. This diagram details the variability constraints of the ECPL at the requirement level. All the constraints represented by this diagram have to be considered during composition to guarantee the overall consistency of the SPL behaviour. The dark grey boxes are features of the ECPL. The light grey boxes are some of the variation points that appear in the FSMr description of the features. Only the variation points involved in SPL level constraint appear. The black arrows are cross-tree constraints. The one from the "Manual" configuration to the "Shift out of park" configuration and to the "Shift into park" configuration says that if the transmission is manual, the targeted configurations cannot be selected. That is, in "Manual" configuration, there is no "park" gear.

The variability constraints of the ECPL at the design level do not contain the cross-tree constraint. The only restriction imposed by this composition constraint is that the FSMds of the features Power lock and Post-crash unlock are ever present. The other FSMd machines can be composed freely.

\section{Modelling features and SPLs}

We use a slightly different notion of feature from the one used in the SPL literature.

In prior works such as in [1], a feature is an incremental function or computation and a system is composed of a core function with an optional list of features. The core function with one or more features satisfying the variability constraints defines the set of all products in the SPL. An important point to be noted is that the system exhibits variability but not the features.

In contrast, our approach considers a feature as a basic unit of functionality and a system is a composition of features. Here, each feature may exhibit variability and the system variability is inherited from that of its constituent features. We adopted this terminology as it is consistent with that of the automotive application domain, which triggered this work. Recent research activities start considering the case of feature with attributes [5].

In this section, we focus on individual features and their modelling. The modelling of the whole system is taken up in 
the next section. As noted in the introduction, we restrict ourselves in this paper to only two levels of abstraction in the development life cycle: the requirement and design levels.

\subsection{Modelling the behaviour of a single feature}

A feature, as mentioned earlier, exhibits variability. In order to describe a feature, we introduce Finite State Machines with Variability (FSMv), an extension of finite state machines (FSMs). Let Var be a finite set of variables, each taking a value ranging over a finite set of values. Given $x \in \operatorname{Var}$, let $\operatorname{Dom}(x)$ denote the finite set of values $x$ can assume. We define $A_{V a r}$ to be the following set of atomic formulas over Var and the values in the domains of variables in Var: $x=a$, $x \neq a$, for $a \in \operatorname{Dom}(x)$, and $x=y, x \neq y$ for $x, y \in \operatorname{Var}$. Define $\Delta::=A_{\text {Var }}|\neg \Delta| \Delta \wedge \Delta|\Delta \vee \Delta| \Delta \rightarrow \Delta \mid$ true $\mid$ false to be the set of all well-formed predicates over Var.

An FSMv is an extension of FSM with the addition of guard (predicate) over the transitions. Guard is specific to transitions, which enables or disables it. A configuration is assignment of values to the variables in Var. Global predicates are consistent, which means they must be satisfied by configurations.

Definition 1 (FSMv) An FSMv is a tuple

$\mathcal{A}=\left\langle Q, q_{0}, \Sigma\right.$, Var $\left., E, \rho\right\rangle$ where:

(1) $Q$ is a finite set of states; $q_{0}$ is the initial state; (2) $\Sigma$ is a finite set of events; (3) Var is a finite set of variables; (4) $E \subseteq Q \times \Delta \times \Sigma \times Q$ gives the set of transitions. A transition $t=\left(s, g, a, s^{\prime}\right)$ represents a transition from state $s$ to state $s^{\prime}$ on event $a$; the predicate $g$ is called a guard of the transition $t ; g$ is defined as the variability domain of the transition; (5) $\rho \in \Delta$ is a consistent predicate called the global predicate.

The variables in Var determine the variability allowed in the feature with each possible valuation of the variables corresponding to a variant. The allowed values of the variables are constrained by the global predicate $\rho ; \rho$ is consistent across all the features of an SPL; $\rho$ are such predicates that do not change across all configurations $\pi$. For example, if $\rho$ is $((x=1) \vee(x=2)) \wedge(y \neq x)$, then the allowed variants are those for which the values for the pairs $(x, y)$ are $(1,2),(2,1)$ (with $y \in\{1,2\})$. The predicate in a transition determines the variants to which the transition is applicable. While drawing a transition $t=\left(s, g, a, s^{\prime}\right)$, the edge connecting $s$ to $s^{\prime}$ is decorated with $g: a$. When $g$ is the predicate "true", we simply write $a$ on the edge.

Definition 2 (Configuration) A configuration, denoted by $\pi$, is an assignment of values to the variables in Var. The set of all configurations is denoted by $\Pi_{V a r}$, or $\Pi$, when Var is clear from the context. Define $\Pi(\rho)=\{\pi \mid \pi \models \rho\}$ to be the set of all those configurations that satisfy $\rho$. The elements of $\Pi(\rho)$ are called valid configurations. Given a valid configuration $\pi$ and a transition $t=\left(s, g, a, s^{\prime}\right)$, we say that $t$ is enabled by $\pi$ if $\pi \models g$.
As a concrete example of an FSMv, consider the feature Door lock in an automotive SPL, which controls the locking of the doors of a car. The expected behaviour of this feature is modelled using the FSMv Reqdl described pictorially in figure 3. The Var for $R e q_{d l}$ in figure 3 is $\quad\left\{\right.$ Transmission $_{d l}=\{$ Auto, Manual $\}, D L \_U s e r \_P r e f=$ $\{$ Speed,Park $\}$ and global predicate $\rho$ is $\{$ Manual $\rightarrow$ Speed $\}$. In the initial state, this feature becomes active when all the doors are closed. The doors are locked when either the speed of the vehicle exceeds a predefined value or the gear is shifted out of park. An unlock event reactivates the feature.

There are three configurations for this feature, all of which are described using the two variables: Transmission $d l$ and DL_User_Pref. To avoid clutter in the diagram, we write any atomic formula, $x=i$, simply as $i$, in the global predicate as well as in guards; $\rho$ ensures that in every valid configuration, the variable Transmission $_{d l}$ having the value Manual implies that DL_User_Pref takes the value Speed. This captures the fact that in manual transmission, there is no park position on the gearbox. The set of events for the state machine is $\{$ AllDoorsClosed, OneDoorClosed, ShiftOutofPark, SpeedUp, Lock, Unlock $\}$. In the state machine diagram, the transition labelled Auto\&Park:ShiftOutofPark denotes the fact that this transition is applicable only when DL_User_Pref is set to Park; and the transmission is automatic. The ShiftOutofPark denotes the event required to trigger this transition.

A configuration allows us to generate various behaviours for a feature from FSMv. A global predicate $\rho$ for a given FSMv restricts a few constraints at feature levels across all the configuration $\pi$, so that FSMv does not generate unrequired behaviour. In door lock example, the vehicles with Manual transmission will always be restricted to car door locking preference as Speed.

Requirement against design: As we can see from the earlier example, in the requirement of a product line, the variability is usually discussed in terms of variation points, like Transmission Type, User Preference, etc. These variation points are at a high level of abstraction and focused on clarity and expressibility. The restriction of the possible configurations is expressed as general constraints on these variation points, e.g., the global predicate Manual $\rightarrow$ Speed in the Door lock example. In contrast, in a design, the variability description is constrained by efficiency,

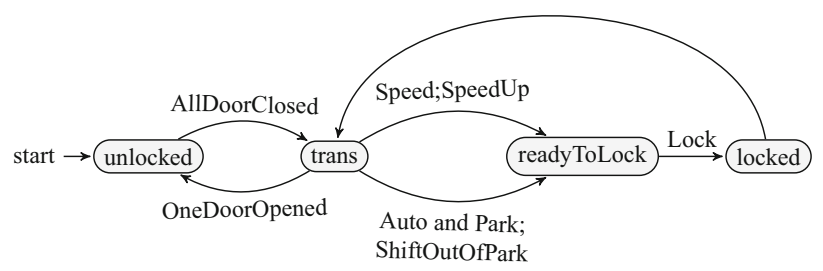

Figure 3. Door lock FSMv. 
implementability, ease of reconfiguration and deployment considerations. For instance, in the automotive applications, one often finds a list of Boolean-valued calibration parameters, which are set appropriately at the time of deployment to obtain a specific variant. Further, the constraint on the calibration parameters $(\rho)$ also takes the special form of the list of the possible configurations of the different calibration parameters in order to easily configure the design. The primary concern in the choice of calibration parameters and their constraints is ease of deployability, which constrains the number of variables used and the type of constraints.

FSMv can capture both the design as well as the requirements of a feature. We distinguish the requirement and design models by denoting them as FSMr and FSMd, respectively. Thus, figure 3 presents the FSMr, $\operatorname{Req}_{d l}$ of the feature Door lock.

Figure 5 describes FSMd, Des $s_{d l}$, the design model of the Door lock feature. The structure of $D e s_{d l}$ is similar to that of $R e q_{d l}$ except that the top elliptical shaped state (trans) in figure 3 is split into two states (transPark and transSpeed) in figure 5. The Var for Des $\operatorname{De}_{d l}$ in figure 5 is $\{C p 1=\{$ Park,Speed $\}\}$. The top state is for ShiftOutOfPark $(\{C p 1=\{$ Park $\}\})$ whereas the bottom one is for SpeedUp $(\{C p 1=\{$ Speed $\}\})$ as can be seen from the configuration label of the two transitions going from the initial state. One major difference to be noted in these two machines is the variability representation. One variable $C p 1$ encodes the possible configurations in the FSMd.

Variants of FSMv and conformance: Having described the design and requirement behaviour of a feature $f$, we now define the notions of variants and conformance. A variant of an FSMv corresponds to one of the several possible behaviours of the feature (at the design and requirement levels).

For a given configuration $\pi$, several transitions from FSMv will be enabled or disabled based on guard (predicates) satisfiability ( $\pi \models g$ ) and the obtained FSM is called as variants of FSMv. If any FSMv does not contain any global predicate $\rho$, then by default it is assumed as true.

Definition 3 (Variant of an FSMv) Let $\mathcal{A}=\left\langle Q, q_{0}, \Sigma\right.$, Var, $\left.E, \rho\right\rangle$ be an FSMv and $\pi \in \Pi(\rho)$ be a valid configuration of $\mathcal{A}$. A variant of $\mathcal{A}$ is the standard FSM obtained from the state machine of $A$, by retaining only the transitions $t=\left(s, g, a, s^{\prime}\right)$, and states $s, s^{\prime}$ such that $\pi \models g$. Once the relevant states and transitions are identified, we remove the guards $g$ from all the transitions. The resultant FSM is denoted $\mathcal{A} \downarrow \pi$.

Figure 4 shows the variant (projection ) for FSMr in figure 3, which is represented as Reqdl $\downarrow\langle$ Manual, Speed $\rangle$. In this variant, the transition with event ShiftOutOfPark is eliminated at it does not satisfy guard condition. Similarly, for the FSMd in figure 5, figure 6 shows the variant, which is represented as $\operatorname{Des}_{d l} \downarrow\langle$ Speed $\rangle$.

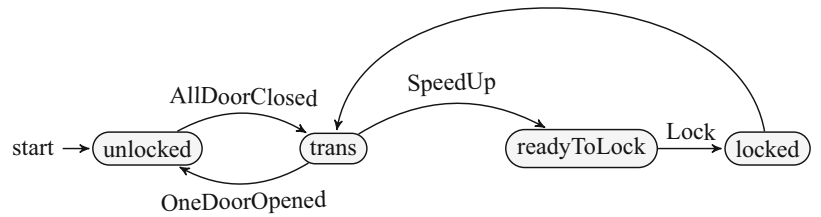

Figure 4. Projection on FSMr for configuration $\pi=$ $\left\{\right.$ Transmission $_{d l}=$ Manual, DL_User_Perf $=$ Speed $\}$.

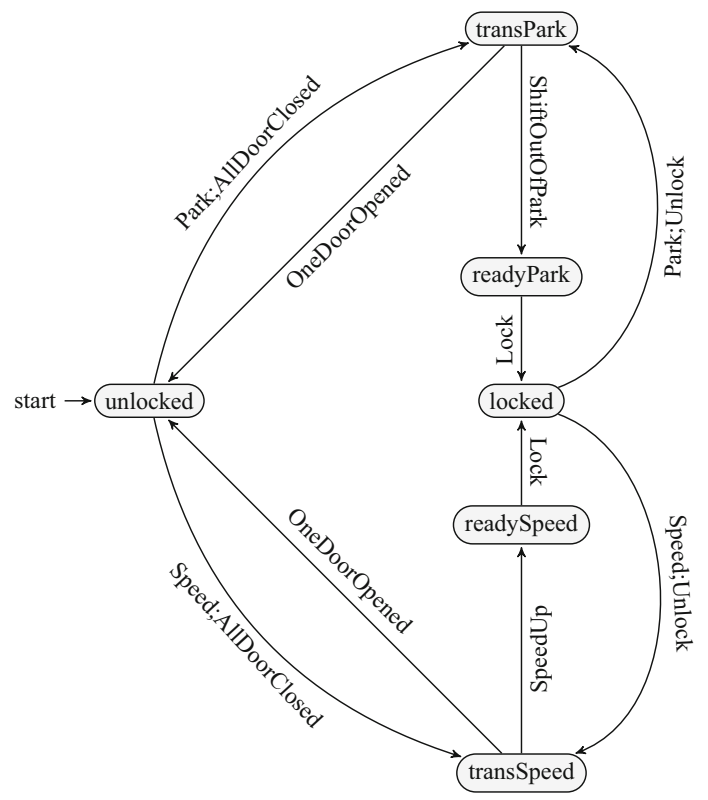

Figure 5. $\operatorname{Des}_{d l}$ : the FSMd abstracted from the design of the feature Door lock.

Given a feature $f$, and a (FSMd, FSMr) pair corresponding to $f$, we define a notion of conformance. We say that the design of $f$ conforms to the requirements of $f$, if and only if every variant of the FSMd has a corresponding FSMr variant. Given an FSMv $\mathcal{A}$, we associate with each configuration $\pi$ of $\mathcal{A}$ the language of the FSM $\mathcal{A} \downarrow \pi$, denoted by $L(\mathcal{A} \downarrow \pi)$. We say that an FSMd $\mathcal{A}_{d}$ conforms to an FSMr $\mathcal{A}_{r}$ if and only if the behaviour of every variant of $\mathcal{A}_{d}$ is contained in the behaviour of some variant of $\mathcal{A}_{r}$.

Definition 4 (The conformance mapping $\Phi$ ) Let $\mathcal{A}_{r}$ and $\mathcal{A}_{d}$ be a pair of FSMr and FSMd, respectively, with global predicates $\rho^{r}$ and $\rho^{d}$. Let $\Pi_{d}, \Pi_{r}$ be the set of all design, requirement configurations. Then $\mathcal{A}_{d}$ conforms to $\mathcal{A}_{r}$ if there exists a mapping $\Phi: \Pi_{d}\left(\rho^{d}\right) \rightarrow 2^{\Pi_{r}\left(\rho^{r}\right)}$ as follows: for any $\pi_{d} \in \Pi_{d}\left(\rho^{d}\right), \quad \Phi\left(\pi_{d}\right)=\left\{\pi_{r} \in \Pi_{r}\left(\rho^{r}\right) \mid L\left(\mathcal{A}_{d} \downarrow \pi_{d}\right) \subseteq\right.$ $\left.L\left(\mathcal{A}_{r} \downarrow \pi_{r}\right)\right\}$. $\Phi$ is called the conformance mapping, and the conformance via $\Phi$ is denoted as $\mathcal{A}_{d} \leq_{\Phi} \mathcal{A}_{r}$.

Conformance check is used to map a projection of FSMd with one of the projections of FSMr for some feature $f$. Such mapping is recorded in $\Phi$. For example, in the feature Door lock, $\Phi(\langle$ Speed $\rangle)=\{\langle$ Manual, Speed $\rangle$, $\langle$ Auto, Speed $\rangle\}$ and $\Phi(\langle$ Park $\rangle)=\{\langle$ Auto, Park $\rangle\}$. Conformance mapping is required to trace any variant in design 


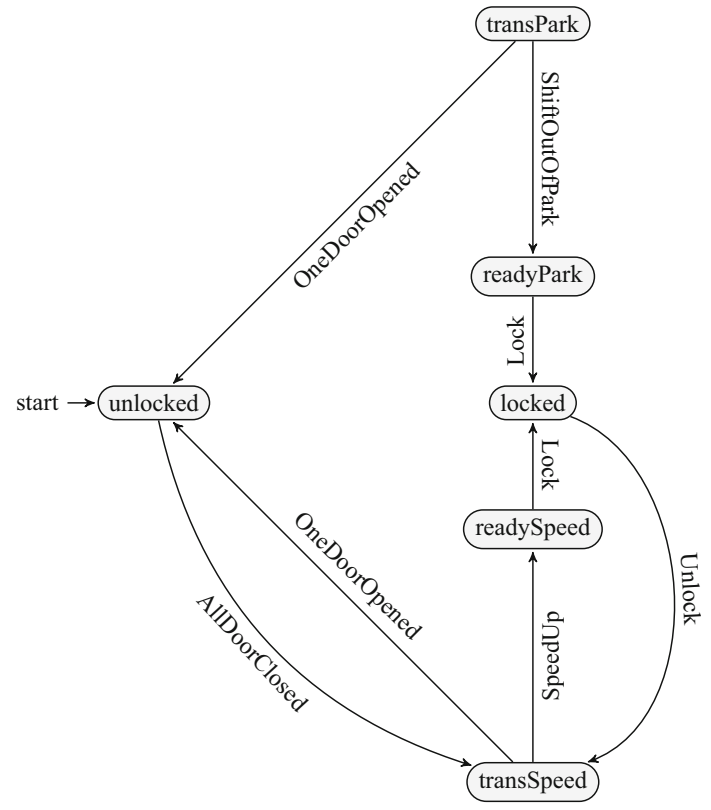

Figure 6. Projection on FSMd for configuration $\pi=\{$ Cp $1=$ Speed $\}$.

that is not mapping to any variant of requirements. In such cases, design is not as per the requirement and must be corrected. We solve the scalability problem of doing conformance check at SPL level by doing conformance check at feature level, and encoding feature level conformance mapping in QBF. This encoded QBF represents equivalent conformance check at SPL level.

\subsection{Modelling the behaviour of an SPL}

In general, an SPL contains multiple features. Each feature may bring its own variability and there could be constraints relating the variabilities among different features. The behaviour of the whole system is a composition of the behaviour of the features. There exists in the literature several kinds of compositions $[10,11]$. Our focus in this paper is variability and SPL modelling and hence we restrict ourselves to one of the well-known compositions, namely synchronous composition. By no means, our study is complete as far as the compositions are concerned. We believe that the issues related to the interaction of variability and composition will be similar to the one discussed here and the present work is a starting point for studying other compositions.

We define a parallel composition operator over FSMv to model an SPL. The features in an SPL can interact and we follow one of the standard methods of allowing the composed FSMv models to share some common events, which correspond to two-party handshake communication events. A distinguishing aspect of the proposed parallel operator is that it takes into account the constraints over variability information across the composed machines.
Definition 5 (Parallel composition of FSMv) Let $\mathcal{A}_{i}=\left\langle Q_{i}, q_{0}^{i}, \Sigma_{i}, \operatorname{Var}_{i}, E_{i}, \rho_{i}\right\rangle, i \in\{1,2\}$ be two FSMv with $\operatorname{Var}_{1} \cap \operatorname{Var}_{2}=\emptyset$. Let $H=\Sigma_{1} \cap \Sigma_{2}$ be the set of handshaking events. Let $\rho_{12}$ be a predicate over $\operatorname{Var}_{1} \cup \operatorname{Var}_{2}$, such that $\rho_{12} \wedge \rho_{1} \wedge \rho_{2}$ is consistent; $\rho_{12}$ is the composition predicate that captures the possible constraints between the variabilities of the two composed features. Let $\rho=\rho_{12} \wedge \rho_{1} \wedge \rho_{2}$.

The parallel composition of $\mathcal{A}_{1}$ and $\mathcal{A}_{2}$ denoted by $\mathcal{A}=\mathcal{A}_{1} \| \mathcal{A}_{2}$ is a tuple $\left\langle Q_{1} \times Q_{2},\left(q_{0}^{1}, q_{0}^{2}\right), \Sigma_{1} \cup \Sigma_{2}, \operatorname{Var}_{1} \cup\right.$ $\left.\operatorname{Var}_{2}, E, \rho\right\rangle$ with transitions defined as follows: consider a state $\left(s_{1}, s_{2}\right) \in Q_{1} \times Q_{2}$, and transitions $\left(s_{1}, g_{1}, a_{1}, s_{1}^{\prime}\right) \in E_{1}$ and $\left(s_{2}, g_{2}, a_{2}, s_{2}^{\prime}\right) \in E_{2}$.

(1) If $a_{1}=a_{2}=a \in H$, define $\left(\left(s_{1}, s_{2}\right), g_{1} \wedge g_{2}, a,\left(s_{1}^{\prime}, s_{2}^{\prime}\right)\right)$ $\in E$, if $g_{1} \wedge g_{2}$ is consistent. This transition is enabled under a valid configuration $\pi \in \Pi(\rho)$, such that $\pi \models g_{1} \wedge g_{2}$.

(2) If $a_{1} \in \Sigma_{1} \backslash H$, define $\left(\left(s_{1}, s_{2}\right), g_{1}, a_{1},\left(s_{1}^{\prime}, s_{2}\right)\right) \in E$. This transition is enabled under valid configurations $\pi$ such that $\pi \models g_{1}$.

(3) If $a_{2} \in \Sigma_{2} \backslash H$, define $\left(\left(s_{1}, s_{2}\right), g_{2}, a_{2},\left(s_{1}, s_{2}^{\prime}\right)\right) \in E$. This transition is enabled under valid configurations $\pi$ such that $\pi \models g_{2}$.

For illustration, consider the feature Door unlock, which automates the unlocking of the doors in a vehicle. Figure 7 gives the FSMr of the feature extracted from the requirements. The Var for $\operatorname{Req}_{d u}$ in figure 7 is $\left\{\right.$ Transmission $_{d u}=$ $\{$ Auto,Manual $\}, \quad D U \_U s e r \_P r e f=\{$ Key,Park $\left.\}\right\}$ and global predicate $\rho$ is $\{$ Manual $\rightarrow K e y\}$. From the initial state, the feature becomes active when the event Lock happens. As soon as either the key is removed from ignition or the gear is shifted to park position, the doors get unlocked and the feature Door unlock becomes inactive. Figure 8 presents the FSMd of the feature Door unlock. The Var for $\operatorname{Des}_{d u}$ in figure 8 is $\{C p 2=\{$ Park, Key $\}\}$. It is quite similar to the requirement except that the active state is split into two: the feature reacts to the ignition Off event in one state, and to the Shift Into Park event in another state.

Let us consider the composition of the two FSMr of the features Door lock and Door unlock. The handshake events between the two features are Lock and Unlock. In the

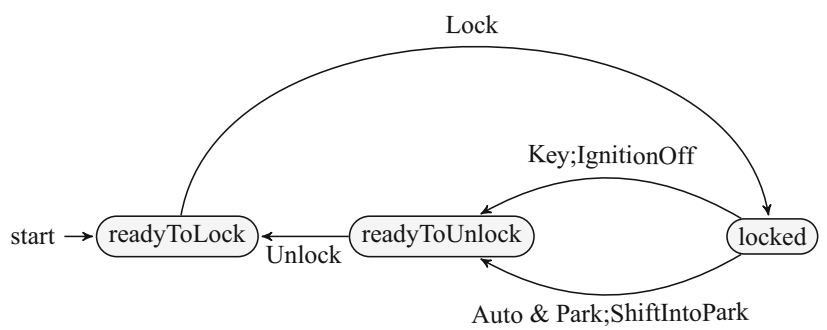

Figure 7. Req $q_{d u}$ : Door unlock FSMr. 


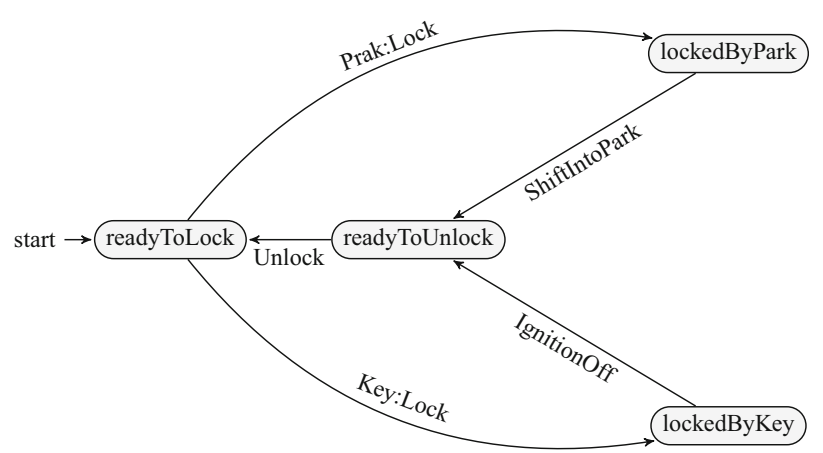

Figure 8. Des ${ }_{d u}$ : Door unlock FSMd.

composition, we introduce the following composition predicate: Transmission $_{d l}=$ Transmission $_{d u}$, which brings out the natural constraints that the transmission status has to be the same. The valid configurations after composition are restricted by the composition predicate. We provide a few definitions to define composite valid configurations.

Definition 6 (Composing Configurations) For $i=1$, 2, let $\mathcal{A}_{i}=\left(Q_{i}, q_{0}^{i}, \Sigma_{i}, \operatorname{Var}_{i}, E_{i}, \rho_{i}\right)$ be two FSMv, and let $\mathcal{A}=\mathcal{A}_{1} \| \mathcal{A}_{2}$ be as given by definition 5. Let $\rho=\rho_{12} \wedge$ $\rho_{1} \wedge \rho_{2}$ be the global predicate of $\mathcal{A}$. Consider two valid configurations $\pi_{1} \in \Pi\left(\rho_{1}\right)$ and $\pi_{2} \in \Pi\left(\rho_{2}\right)$ of $\mathcal{A}_{1}$ and $\mathcal{A}_{2}$. The composition of $\pi_{1}, \pi_{2}$ denoted $\pi_{1}+\pi_{2}$ is a configuration over $V a r_{1} \cup \operatorname{Var}_{2}$ such that (i) $\pi_{1}+\pi_{2}$ agrees with $\pi_{1}$ over $V a r_{1}$, agrees with $\pi_{2}$ over $\operatorname{Var}_{2}$, and (ii) $\pi_{1}+\pi_{2} \models \rho$.

Lemma 7 Let $\mathcal{A}_{1}$ and $\mathcal{A}_{2}$ be two FSMv. For each valid configuration $\pi$ of $\mathcal{A}_{1} \| \mathcal{A}_{2}$, there are valid configurations $\pi_{1}$ of $\mathcal{A}_{1}$ and $\pi_{2}$ of $\mathcal{A}_{2}$ such that $\pi=\pi_{1}+\pi_{2}$.

Proof 1 Let $\pi \in \Pi(\rho)$ with $\rho=\rho_{12} \wedge \rho_{1} \wedge \rho_{2}$ be a valid configuration of $\mathcal{A}_{1} \| \mathcal{A}_{2} ; \rho_{1}$ and $\rho_{2}$ are the global predicates of $\mathcal{A}_{1}$ and $\mathcal{A}_{2}$, respectively, and $\rho_{12}$ is the composition predicate of $\mathcal{A}_{1}, \mathcal{A}_{2}$. By definition of valid configuration, $\pi \models \rho$; hence $\pi \models \rho_{1}$ and $\pi \models \rho_{2}$. Since $\pi$ is a configuration over $\operatorname{Var}_{1} \cup \operatorname{Var}_{2}$, let us consider the restriction of $\pi$ on $\operatorname{Var}_{1}$, call the resulting configuration $\pi_{1}$. Then $\pi_{1} \models \rho_{1}$. Similarly, call the restriction of $\pi$ on $\operatorname{Var}_{2}$ as $\pi_{2}$. Then $\pi_{2} \models \rho_{2}$. Then, $\pi_{1}$ and $\pi_{2}$ are, respectively, valid configurations of $\mathcal{A}_{1}$ and $\mathcal{A}_{2}$. Hence, by definition 6 , we obtain $\pi=\pi_{1}+\pi_{2}$.

In the example of feature Door Lock, the configuration $\langle$ Auto, Speed $\rangle$ from Req $q_{d l}$ can be composed with $\langle$ Auto, Key $\rangle$ from Reqdu because the transmission is Auto in both (which is specified in the composition predicate Transmission $_{d l}=$ Transmission $\left._{d u}\right) .\langle$ Auto, Speed, Auto, Key $\rangle$ is a configuration of the parallel composition of $R e q_{d l}$ with $R e q_{d u}$. The parallel composition of FSMv is such that each variant of the composition of two FSMv is equal to the composition of variants of the individual FSMv.
Lemma 8 (Variants of a composed FSMv) Let $\mathcal{A}_{1}$ and $\mathcal{A}_{2}$ be two FSMv. Let $\pi$ be a valid configuration of $\mathcal{A}_{1} \| \mathcal{A}_{2}$. Then $L\left(\left[\mathcal{A}_{1} \| \mathcal{A}_{2}\right] \downarrow \pi\right)=L\left(\mathcal{A}_{1} \downarrow \pi\right) \| L\left(\mathcal{A}_{2} \downarrow \pi\right)$. ${ }^{1}$

We review some preliminary definitions before the proof. In the following, we denote by the same $\|$, the following operations: (i) shuffle of words, (ii) shuffle of languages, (iii) parallel composition of FSMs and (iv) parallel composition of FSMv. The context would be clear in each case, avoiding any confusion.

Definition 9 Let $\Sigma_{1}, \ldots, \Sigma_{n}$ be $n$ finite sets of symbols. Let $\Sigma$ be a finite set. Given a word $w \in \Sigma^{*}$, we denote by $w \downarrow \Sigma_{i}$ the unique subword of $w$ over $\Sigma_{i}^{*}$. For example, if $\Sigma_{1}=\{a, b, e\}, \Sigma_{2}=\{a, e, f\}, \quad$ and if we consider $w=$ aefedefr $\in\{a, d, e, f, r\}^{*}$, then $w \downarrow \Sigma_{1}=$ aeee $\quad$ and $w \downarrow \Sigma_{2}=$ aefeef .

Definition 10 (Asynchronous shuffle) Let $\Sigma_{1}, \ldots, \Sigma_{n}$ be $n$ finite sets. Let $\Sigma=\cup_{i=1}^{n} \Sigma_{i}$. Consider $n$ words $u_{1}, u_{2}, \ldots, u_{n}, u_{i} \in \Sigma_{i}^{*}$. The asynchronous shuffle of $u_{1}, \ldots, u_{n}$ denoted $u_{1}\|\ldots\| u_{n}$ is defined as $\left\{w \mid w \downarrow \Sigma_{i}=u_{i}\right\}$.

As an example, consider $\Sigma_{1}=\{a, b, c, f\}, \Sigma_{2}=$ $\{a, d, e, f\}, \Sigma_{3}=\{c, d, f\} \quad$ and the words $u_{1}=a b c f, u_{2}=a d f e, u_{3}=d c f$. Then the word $w=a b d c f e$ is in $u_{1}\left\|u_{2}\right\| u_{3}$, since $w \downarrow \Sigma_{i}=u_{i}$ for $i=1,2,3$. Similarly, the word $w^{\prime}=a d b c f e$ is also in $u_{1}\left\|u_{2}\right\| u_{3}$. However, the word $w^{\prime \prime}=a e b c f d$ is not in $u_{1}\left\|u_{2}\right\| u_{3}$, since $w^{\prime \prime} \downarrow \Sigma_{2}=$ aefd, not $u_{2}$.

The definition of shuffle can be extended from words to languages. We use the same notation $\|$ for the shuffle of sets, as well as for the shuffle of words.

The asynchronous shuffle of two languages $L_{1}, L_{2}$ is defined as $L_{1} \| L_{2}=\left\{w_{1} \| w_{2} \mid w_{1} \in L_{1}, w_{2} \in L_{2}\right\}$. For example, if $L_{1}=\{a b c f, a b b f\}$ is a language over $\Sigma_{1}=\{a, b, c, f\}$ and $L_{2}=\{a d f e\}$ is a language over $\{a, d, e, f\}$, then $L_{1} \| L_{2}=\{a b c f \|$ adfe, abbf $\|$ adfe $\}$ $=\{a b c d f e$, adbcfe, abdcfe, abbdfe, abdbfe, adbbfe $\}$.

Definition 11 (Asynchronous product) Let $M_{i}=$ $\left(Q_{i}, q_{i}, \Sigma_{i}, \delta_{i}\right)$ and $M_{j}=\left(Q_{j}, q_{j}, \Sigma_{j}, \delta_{j}\right)$ be complete FSMs. The asynchronous product of $M_{i}, M_{j}$ is defined as the FSM $M_{i} \| M_{j}=\left(Q_{i} \times Q_{j},\left(q_{i}, q_{j}\right), \Sigma_{i} \cup \Sigma_{j}, \delta\right)$ where

1. $\delta\left(\left(q, q^{\prime}\right), a\right)=\left(\delta_{i}(q, a), \delta_{j}\left(q^{\prime}, a\right)\right), a \in \Sigma_{i} \cap \Sigma_{j}$,

2. $\delta\left(\left(q, q^{\prime}\right), a\right)=\left(\delta_{i}(q, a), q^{\prime}\right), a \in \Sigma_{i}, a \notin \Sigma_{j}$,

3. $\delta\left(\left(q, q^{\prime}\right), a\right)=\left(q, \delta_{j}\left(q^{\prime}, a\right)\right), a \in \Sigma_{j}, a \notin \Sigma_{i}$.

On the common events, both FSMs move in parallel; otherwise, they move independent of each other.

Proof 2 of Lemma 8 It is known that $L\left(M_{i} \| M_{j}\right)=$ $L\left(M_{i}\right) \| L\left(M_{j}\right)$. Consider a valid configuration $\pi$ of $\mathcal{A}_{1} \| \mathcal{A}_{2}$. As seen in Lemma 7 , we can find valid

\footnotetext{
${ }^{1}$ The right hand side of $\|$ refers to the standard communicating FSM composition.
} 
configurations $\pi_{1}$ of $\mathcal{A}_{1}$ and $\pi_{2}$ of $\mathcal{A}_{2}$ such that $\pi=\pi_{1}+\pi_{2}$. The initial state of $\mathcal{A}_{1} \| \mathcal{A}_{2}$ is $\left(q_{0}^{1}, q_{0}^{2}\right)$, where $q_{1}^{0}$ is the initial state of $\mathcal{A}_{1}$ and $q_{2}^{0}$ is the initial state of $\mathcal{A}_{2}$. By definitions 5 and 11 , if we consider a string $w=a_{1} a_{2} \ldots a_{n} \in L\left[\mathcal{A}_{1} \| \mathcal{A}_{2}\right] \downarrow \pi$, then we can find strings $w_{1} \in L\left(\mathcal{A}_{1} \downarrow \pi\right)=L\left(\mathcal{A}_{1} \downarrow \pi_{1}\right) \quad$ and $\quad w_{2} \in L\left(\mathcal{A}_{2} \downarrow \pi\right)=$ $L\left(\mathcal{A}_{2} \downarrow \pi_{2}\right)$ such that $w$ can be written as an asynchronous shuffle of $w_{1}$ and $w_{2}$ in the sense of definition 10. Hence, $L\left[\mathcal{A}_{1} \| \mathcal{A}_{2}\right] \downarrow \pi \subseteq L\left(\mathcal{A}_{1} \downarrow \pi\right) \| L\left(\mathcal{A}_{2} \downarrow \pi\right)$. The converse can be shown in a similar way.

Refinement and parallel composition: The definition of parallel composition naturally lends itself to a notion of addition of conformance mappings between design and requirement pairs. Consider FSMr $R_{1}, R_{2}$ corresponding to two features $f_{1}, f_{2}$. Let $D_{1}, D_{2}$ be the corresponding FSMd. Let $\rho_{1}^{r}, \rho_{2}^{r}$ be the global predicates of $R_{1}, R_{2}$, and let $\rho_{1}^{d}, \rho_{2}^{d}$ be the global predicates of $D_{1}, D_{2}$, respectively. Assume that $D_{1} \leq \Phi_{1} R_{1}$ and $D_{2} \leq \Phi_{\Phi_{2}} R_{2}$. Let $\rho^{r}=\rho_{12}^{r} \wedge \rho_{1}^{r} \wedge \rho_{2}^{r}$ be the global predicate of $R_{1} \| R_{2}$; likewise, let $\rho^{d}=\rho_{12}^{d} \wedge$ $\rho_{1}^{d} \wedge \rho_{2}^{d}$ be the global predicate of $D_{1} \| D_{2}$. We now want to ask if $D_{1} \| D_{2}$ conforms to $R_{1} \| R_{2}$. This amounts to computing a conformance mapping between $D_{1} \| D_{2}$ and $R_{1} \| R_{2}$ given $\Phi_{1}, \Phi_{2}$. Consider any valid configuration $\pi^{d}$ of $D_{1} \| D_{2}$. By Lemma 7 , we can write $\pi^{d}$ as $\pi_{1}^{d}+\pi_{2}^{d}$, where $\pi_{1}^{d}$ and $\pi_{2}^{d}$ are valid configurations of $D_{1}$ and $D_{2}$, respectively. Since $D_{1} \leq \Phi_{1} R_{1}$ and $D_{2} \leq{ }_{\Phi_{2}} R_{2}$, there exist valid configurations $\pi_{1}^{r} \in \Phi_{1}\left(\pi_{1}^{d}\right)$ and $\pi_{2}^{r} \in \Phi_{2}\left(\pi_{2}^{d}\right)$ in $R_{1}$ and $R_{2}$, respectively. Given this, the addition of $\Phi_{1}, \Phi_{2}$ is defined in definition 12 .

Definition 12 (Addition of conformance mappings) The addition of conformance mappings $\Phi_{1}, \Phi_{2}$ is defined to be a mapping $\Phi=\Phi_{1}+\Phi_{2}$ as follows. For every valid configuration $\pi^{d}=\pi_{1}^{d}+\pi_{2}^{d}$ of $D_{1} \| D_{2}$,

$\Phi\left(\pi^{d}\right)=\left\{\pi^{r} \mid \pi^{r}\right.$ is a valid configuration of $R_{1} \| R_{2}, \pi^{r}=$ $\pi_{1}^{r}+\pi_{2}^{r}$ for valid configurations $\left.\pi_{1}^{r} \in \Phi_{1}\left(\pi_{1}^{d}\right), \pi_{2}^{r} \in \Phi_{2}\left(\pi_{2}^{d}\right)\right\}$

Note that by definition $12, \Phi$ could be empty. Consider a valid configuration $\pi^{d}=\pi_{1}^{d}+\pi_{2}^{d}$ of $D_{1} \| D_{2}$. If there is no valid configuration $\pi^{r}$ of $R_{1} \| R_{2}$ that is a composition of valid configurations $\pi_{1}^{r} \in \Phi_{1}\left(\pi_{1}^{d}\right), \pi_{2}^{r} \in \Phi_{2}\left(\pi_{2}^{d}\right)$, then $\Phi$ is empty (or there is no conformance mapping $\Phi$ between $D_{1} \| D_{2}$ and $R_{1} \| R_{2}$ ). If $\Phi$ exists, then we can say the following.

Lemma 13 (Conformance of composition) Let $R_{1}$ and $R_{2}$ be two FSMr corresponding to features $f_{1}, f_{2}$, and let $D_{1}$ and $D_{2}$ be the corresponding FSMds. Let $D_{1} \leq \Phi_{1} R_{1}$ and $D_{2} \leq \Phi_{2} R_{2}$. Let $\Phi=\Phi_{1}+\Phi_{2}$ and $\pi^{d}$ be a valid configuration of $\quad D_{1} \| D_{2}$. Then, $\forall \pi^{r} \in \Phi\left(\pi^{d}\right)$, $L\left(\left[\left(D_{1} \| D_{2}\right) \downarrow \pi^{d}\right]\right) \subseteq L\left(\left[\left(R_{1} \| R_{2}\right) \downarrow \pi^{r}\right]\right)$.

Proof 3 Given a valid configuration $\pi^{d}$ of $D_{1} \| D_{2}$, we can write it as $\pi_{1}^{d}+\pi_{2}^{d}$, where $\pi_{1}^{d}, \pi_{2}^{d}$ are, respectively, valid configurations of $D_{1}, D_{2}$ (Lemma 7). Since $D_{1} \leq{ }_{\Phi_{1}} R_{1}$ and
$D_{2} \leq{ }_{\Phi_{2}} R_{2}$, there exist valid configurations $\pi_{1}^{r} \in \Phi_{1}\left(\pi_{1}^{d}\right)$ and $\pi_{2}^{r} \in \Phi_{2}\left(\pi_{2}^{d}\right) \quad$ such that $L\left(D_{1} \downarrow \pi_{1}^{d}\right) \subseteq L\left(R_{1} \downarrow \pi_{1}^{r}\right)$ and $L\left(D_{2} \downarrow \pi_{2}^{d}\right) \subseteq L\left(R_{2} \downarrow \pi_{2}^{r}\right)$.

Since $\Phi$ has been computed, for every valid configuration $\pi^{d}$ of $D_{1} \| D_{2}$, there exists some valid configuration $\pi^{r} \quad$ of $\quad R_{1} \| R_{2}, \quad \pi^{r} \in \Phi\left(\pi^{d}\right)$. As $\pi^{r} \quad$ is valid, $\pi^{r} \models \rho_{12}^{r} \wedge \rho_{1}^{r} \wedge \rho_{2}^{r}$; hence, $\pi^{r}$ can be written as $\pi_{1}^{r}+\pi_{2}^{r}$, where $\pi_{1}^{r}, \pi_{2}^{r}$ are, respectively, valid configurations of $R_{1}, R_{2}$ (Lemma 7), and $\pi_{1}^{r} \in \Phi_{1}\left(\pi_{1}^{d}\right), \quad \pi_{2}^{r} \in \Phi_{2}\left(\pi_{2}^{d}\right)$ by definition 12 .

$L\left(\left[\left(D_{1} \| D_{2}\right) \downarrow \pi^{d}\right]\right)=L\left(D_{1} \downarrow \pi_{1}^{d}\right) \| L\left(D_{2} \downarrow \pi_{2}^{d}\right) \quad$ by lemma 8. Similarly, $L\left(\left[\left(R_{1} \| R_{2}\right) \downarrow \pi^{r}\right]\right)=L\left(R_{1} \downarrow \pi_{1}^{r}\right) \|$ $L\left(R_{2} \downarrow \pi_{2}^{r}\right)$. This along with the observation that $L\left(D_{1} \downarrow\right.$ $\left.\pi_{1}^{d}\right) \subseteq L\left(R_{1} \downarrow \pi_{1}^{r}\right) \quad$ and $\quad L\left(D_{2} \downarrow \pi_{2}^{d}\right) \subseteq L\left(R_{2} \downarrow \pi_{2}^{r}\right) \quad$ gives $L\left(\left[\left(D_{1} \| D_{2}\right) \downarrow \pi^{d}\right]\right) \subseteq L\left(\left[\left(R_{1} \| R_{2}\right) \downarrow \pi^{r}\right]\right)$.

Let us remind that in the feature Door lock, $\Phi(\langle$ Park $\rangle)=$ $\{\langle$ Auto, Park $\rangle\}$ and $\Phi(\langle$ Speed $\rangle)=\{\langle$ Manual, Speed $\rangle$, $\langle$ Auto, Speed $\rangle\}$. Also, in the feature Door unlock, $\Phi^{\prime}(\langle$ Key $\rangle)=\{\langle$ Manual, Key $\rangle, \quad\langle$ Auto, Key $\rangle\} \quad$ and $\Phi^{\prime}(\langle$ Park $\rangle)=\{\langle$ Auto, Park $\rangle\}$. Note that the conformance mapping of Door lock is noted as $\Phi$ whereas the one of Door unlock is noted as $\Phi^{\prime}$.

In the FSMr Req $q_{d l} \| R e q_{d u}$ with $\rho_{r}:$ Transmission $_{d l}=$ Transmission $_{d u}$. Any configuration with Transmission $d l=$

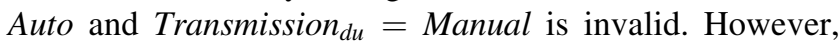
$\Phi(\langle$ Park $\rangle)$ contains a single configuration where Transmission $_{d l}=$ Auto and $\Phi^{\prime}(\langle$ Key $\rangle)$ contains a configuration where Transmission $_{d u}=$ Manual and $\Phi(\langle$ Park $\rangle)+$ $\Phi^{\prime}(\langle K e y\rangle)$ is a valid configuration of $\operatorname{Des}_{d l} \| \operatorname{Des}_{d u}$ with $\rho_{d}:$ true.

Does the design conform to the requirement? Yes because $\Phi^{\prime}(\langle K e y\rangle)$ contains another configuration where Transmission $_{d u}=$ Auto and $\Phi(\langle$ Park $\rangle)+\Phi^{\prime}(\langle$ Key $\rangle)$ is not an empty set:

$\Phi(\langle$ Park $\rangle)+\Phi^{\prime}(\langle$ Key $\rangle)=\{\langle$ Auto, Park, Auto, Key $\rangle\}$. Similarly, $\Phi(\langle$ Park $\rangle)+\Phi^{\prime}(\langle$ Park $\rangle)=\{\langle$ Auto, Park, Auto, Park $\rangle\}, \Phi(\langle$ Speed $\rangle)+\Phi^{\prime}(\langle$ Park $\rangle)=\{\langle$ Auto, Speed, Auto, Park $\rangle\}$, and $\Phi(\langle$ Speed $\rangle)+\Phi^{\prime}(\langle$ Key $\rangle)=\{\langle$ Auto, Speed, Auto, Key $\rangle,\langle$ Manual, Speed, Manual, Key $\rangle$.

It may appear that the addition of conformance mappings leads to an invalid design configuration, i.e., a design configuration of the composed machine that does not conform to any requirement configuration. Assume a variant of the ECPL case study where in the feature Door unlock, $\Phi(\langle$ Speed $\rangle)=\{\langle$ Manual, Speed $\rangle\} \quad$ and $\Phi(\langle$ Park $\rangle)=$ $\{\langle$ Auto, Park $\rangle\}$. Also, in the feature Door unlock, $\Phi^{\prime}(\langle$ Key $\rangle)=\{\langle$ Manual, Key $\rangle\} \quad$ and $\quad \Phi^{\prime}(\langle$ Park $\rangle)=$ $\{\langle$ Auto, Park $\rangle\}$.

In this hypothetical case, $\Phi(\langle$ Speed $\rangle)+\Phi^{\prime}(\langle$ Park $\rangle)$ and $\Phi(\langle$ Park $\rangle)+\Phi^{\prime}(\langle$ Key $\rangle)$ are empty because the corresponding requirement configurations are incompatible with respect to the composition constraint: Transmission $_{d l}=$ Auto and Transmission Tu $_{\text {Tanual. }}$. 
Hence, the design does not conform to the requirement. However, if we consider the composition predicate $\rho_{d}: C p 1=$ Park $\Leftrightarrow C p 2=$ Park, then $\langle$ Speed $\rangle$ and $\langle$ Park $\rangle$ are not compatible anymore $(\langle P a r k\rangle$ and $\langle$ Key $\rangle$ neither $)$ and as a result the design conforms to the requirement: $\Phi(\langle$ Park $\rangle)+\Phi^{\prime}(\langle$ Park $\rangle)=\{\langle$ Auto, Park, Auto, Park $\rangle\}$ and $\Phi(\langle$ Speed $\rangle)+\Phi^{\prime}(\langle$ Key $\rangle)=\{\langle$ Manual, Speed, Manual, Key $\rangle\}$.

\section{Feature level verification}

In section 2, we introduced FSMv for modelling the behaviour of a feature and illustrated how they can express variability at two different levels of description. We also defined a conformance relation to formally relate the variabilities at two different levels of abstraction. In this section, we will give an automatic procedure for checking the conformance relation.

Let $f$ be a feature whose requirement and design level descriptions are given by FSMr $R e q_{f}$ and FSMd $D e s_{f}$, respectively. The proposed verification method, directly following the definition of conformance, computes a mapping $\Phi$ such that $D e s_{f} \leq_{\Phi} R e q_{f}$. A straightforward approach to computation is to identify, for each design configuration, one or more requirement configurations. The conformance checking fails if there does not exist any requirement configuration for a design configuration.

Algorithm 1, given later, presents a possible implementation using the standard automata containment algorithm [12], as implemented in the well-known model checking tool SPIN [8]. Algorithm 1 runs the full verification algorithm of SPIN for every pair $\left(\pi_{d}, \pi_{r}\right)$ of design and requirement configurations. SPIN returns the list of pairs for which the conformance condition is violated. Every other pair is added to the conformance mapping $\Phi$.

Lemma 14 proves the correctness of Algorithm 1.

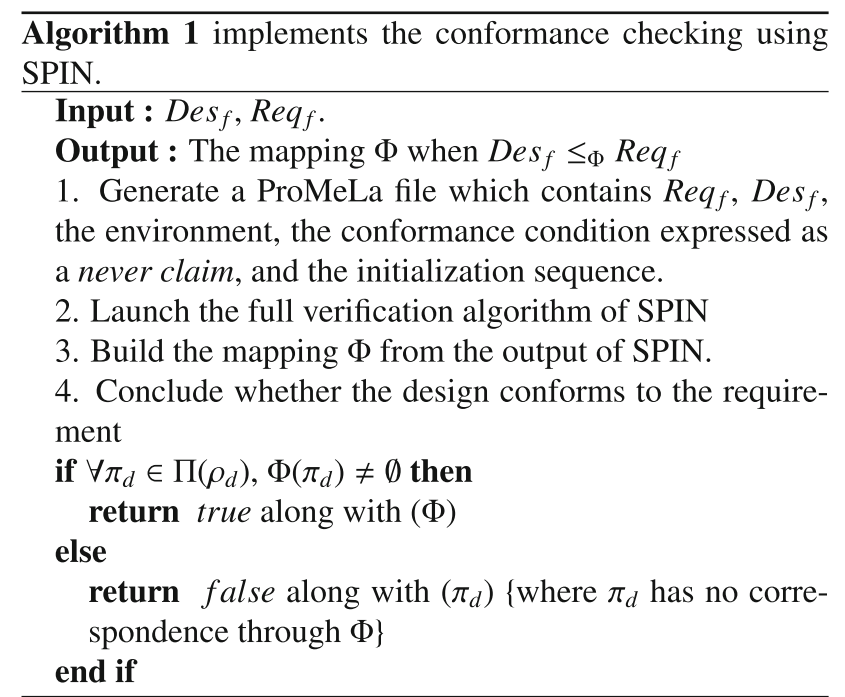

Lemma 14 Given FSMd Des $f$ and FSMr Req for a feature $f$, let $\left(\pi_{d}, \pi_{r}\right)$ be a pair of design and requirement configurations. Then, $L\left(\operatorname{Des}_{f} \downarrow \pi_{d}\right) \nsubseteq L\left(\operatorname{Req}_{f} \downarrow \pi_{r}\right)$ if and only if $\neg \operatorname{error}\left(\operatorname{Des}_{f}\right) \wedge \operatorname{error}\left(\operatorname{Req}_{f}\right)$.

Proof 4 Assume $L\left(\operatorname{Des}_{f} \downarrow \pi_{d}\right) \nsubseteq L\left(\operatorname{Req}_{f} \downarrow \pi_{r}\right)$. Then there exists a word $w \in L\left(\operatorname{Des}_{f} \downarrow \pi_{d}\right)$ that is prefixed by u.e, with $u$ a finite prefix of a word in $L\left(R e q_{f} \downarrow \pi_{r}\right)$, and $e$ an event such that u.e is not a prefix of any word in $L\left(R e q_{f} \downarrow \pi_{r}\right)$. In such a situation, Des does not go to the error state but $R e q_{f}$ does.

Conversely, if $L\left(\operatorname{Des}_{f} \downarrow \pi_{d}\right) \subseteq L\left(R e q_{f} \downarrow \pi_{r}\right)$, then whenever $D e s_{f}$ is not in an error state, $R e q_{f}$ will also not be in an error state.

The formula $\neg \operatorname{error}\left(\operatorname{Des}_{f}\right) \wedge \operatorname{error}\left(\operatorname{Req}_{f}\right)$ is true when $L\left(\operatorname{Des}_{f} \downarrow \pi_{d}\right) \nsubseteq L\left(\operatorname{Req}_{f} \downarrow \pi_{r}\right)$. When a word $w \in L\left(\operatorname{Des}_{f} \downarrow\right.$ $\left.\pi_{d}\right)$ and $w \notin L\left(\operatorname{Req}_{f} \downarrow \pi_{r}\right)$ then $\operatorname{error}\left(\operatorname{Des}_{f}\right)$ is false and $\operatorname{error}\left(\operatorname{Req}_{f}\right)$ is true. This make $L\left(\operatorname{Des}_{f} \downarrow \pi_{d}\right) \nsubseteq L\left(\operatorname{Req}_{f} \downarrow \pi_{r}\right)$ true, which means the variants design does not conform to the variant of requirement.

It must be noted that we exhaustively check whether every variant of the design conforms to some variant of the requirement. Our experience with real systems is that the number of variants at the feature level is very small. Our experimental results (see section 5) shows that our approach scales well in realistic applications.

\section{System level variability verification}

In this section, we extend the variability verification to a system consisting of two or more features. Let us consider the case when we have $n$ features $f_{1}, f_{2}, \ldots, f_{n}$, whose designs and requirements are described by FSMd $D_{1}, D_{2}, \ldots, D_{n}$ and FSMr $R_{1}, R_{2}, \ldots R_{n}$, respectively. Further, let $D_{i} \leq{ }_{\Phi_{i}} R_{i}$ for $1 \leq i \leq n$. Following Lemma 14, we can check that for each valid design configuration $\pi_{D_{1}\|\ldots\| D_{n}}$ of $D_{1}\|\ldots\| D_{n}$, there exists a configuration $\pi_{R_{1}\|\ldots\| R_{n}}$ of $R_{1}\|\ldots\| R_{n}$ such that $\pi_{R_{1}\|\ldots\| R_{n}}$ is a composition of valid requirement configurations computed via $\Phi_{1}, \ldots, \Phi_{n}$. Then, $D_{1}\|\ldots\| D_{n}$ conforms to $R_{1}\|\ldots\| R_{n}$ via a conformance mapping $\Phi$ that is nothing but $\Phi_{1}+\ldots+\Phi_{n}$. The existence of conformance mapping can be shown by checking that $\Phi$ is non empty. This check is formulated naturally as satisfiability of a quantified Boolean formula.

QBF formulation: Given FSMd $D_{1}, \ldots, D_{n}$ and FSMr $R_{1}, \ldots, R_{n}$, (1) let $\operatorname{Var}\left(D_{i}\right)=\left\{x_{i 1}, \ldots, x_{i d_{i}}\right\}$ be the set of variables of design $D_{i}$, and $\operatorname{Var}\left(R_{i}\right)=\left\{y_{i 1}, \ldots, y_{i r_{i}}\right\}$ the set of variables of requirement $R_{i}$. Let $\pi_{i}^{d}:\left(x_{i 1}=a_{1}, \ldots, x_{i d_{i}}=\right.$ $a_{d_{i}}$ ) be a configuration of $D_{i}$. We denote this by $\pi_{i}^{d}\left(x_{i 1}, \ldots, x_{i d_{i}}\right)$, which is the conjunction $\bigwedge_{l=1}^{d_{i}}\left(x_{i l}=a_{l}\right)$.

(2) Given $n$ FSMd and $n$ FSMr, check if $D_{i}$ conforms to $R_{i}$ for all $1 \leq i \leq n$ using Algorithm 1. This gives the map $\Phi_{i}$. Assume $D_{i}$ has $m$ distinct configurations 
$\pi_{i 1}^{d}, \ldots, \pi_{i m}^{d}$. For $1 \leq j \leq m$, let $\Phi_{i}\left(\pi_{i j}^{d}\right)=\left\{\pi_{i j_{1}}^{r}, \ldots, \pi_{i j_{k}}^{r}\right\}$, where each item of $\pi_{i j_{1}}^{r}, \ldots, \pi_{i j_{k}}^{r}$ is a configuration of $R_{i}$, which has been mapped by $\Phi_{i}$ to some configuration $\pi_{i j}^{d}$ of $D_{i} . \quad \Phi_{i}\left(\pi_{i j}^{d}\right)$ can be written as the formula $\pi_{i j_{1}}^{r} \vee \ldots \vee \pi_{i j_{k}}^{r}$.

(3) The conformance mapping $\Phi_{i}$ between $D_{i}$ and $R_{i}$ has the form $\bigwedge_{j=1}^{m} \Phi_{i}\left(\pi_{i j}^{d}\right)$. (4) Let $\varphi_{i, j}^{d}=\rho^{d} \wedge \rho_{i}^{d} \wedge \rho_{j}^{d}$ and $\varphi_{i, j}^{r}=$ $\rho^{r} \wedge \rho_{i}^{r} \wedge \rho_{j}^{r}$ represent, respectively, the propositional formula that ensures the consistency of the global predicates of $D_{i}, D_{j}$ and $R_{i}, R_{j}$ along with the compositional predicates $\rho^{d}$ and $\rho^{r}$. Given a set $S \subseteq\{1,2, \ldots, n\}$, $\varphi_{S}^{d}$ and $\varphi_{S}^{r}$ can be appropriately written.

The global predicate $\rho$ can be at feature level and also at SPL level. When we do feature level conformance, then feature level $\rho$ must be satisfied by the configuration $\pi$. When we do SPL level conformance, then all feature level $\rho$ plus SPL level $\rho$ must be satisfied.

The QBF for conformance checking is given by $\Psi=\forall x_{11} \ldots x_{1 d_{1}} x_{21} \ldots x_{2 d_{2}} \ldots x_{n 1} \ldots x_{n d_{n}}\left[\varphi_{1,2, \ldots, n}^{d} \Rightarrow \exists y_{11}\right.$ $\left.\ldots y_{1 r_{1}} y_{21} \ldots y_{2 r_{2}} \ldots y_{n 1} \ldots y_{n r_{n}}\left(\Phi_{1} \wedge \ldots \wedge \Phi_{n} \wedge \varphi_{1,2, \ldots, n}^{r}\right)\right]$

Theorem 15 asserts that the QBF $\Psi$ holds iff a conformance mapping $\Phi$ exists such that $D_{1}\|\ldots\| D_{n} \leq{ }_{\Phi} R_{1}\|\ldots\| R_{n}$.

Theorem 15 Given an SPL, let $\left\{f_{1}, \ldots, f_{n}\right\}$ be the set of features in a chosen product. Let $D_{i}, R_{i}$ be the FSMd and FSMr for feature $f_{i}$. Then $D_{1}\|\ldots\| D_{n}$ conforms to $R_{1} \|$ ... $\| R_{n}$ iff $\Psi$, as defined earlier, is satisfiable.

Proof 5 Given $D_{i} \leq \Phi_{i} R_{i}$, for $1 \leq i \leq n$, assume that $D_{1} \|$ ... $\| D_{n}$ conforms to $R_{1}\|\ldots\| R_{n}$. Then, by definition of conformance, for all valid configurations $\pi^{d}$ of $D_{1}\|\ldots\| D_{n}$, there exists a valid configuration $\pi^{r}$ of $R_{1} \|$ $\ldots \| R_{n} \quad$ such that $L\left(\left[D_{1}\|\ldots\| D_{n}\right] \downarrow \pi^{d}\right) \subseteq L\left(\left[R_{1}\right.\right.$ $\left.\left.\|\ldots\| R_{n}\right] \downarrow \pi^{r}\right)$. Let $\Phi$ be the conformance mapping such that $\pi^{r} \in \Phi\left(\pi^{d}\right)$.

$\pi^{d}$ is a valid configuration of $D_{1}\|\ldots\| D_{n}$ implies that $\pi^{d} \models \bigwedge_{S \subseteq\{1,2, \ldots, n\}} \rho_{S}^{d}$, where $\rho_{S}^{d}$ is the global predicate of $D_{i_{1}}\|\ldots\| D_{i_{j}}$, when $S=\left\{i_{1}, \ldots, i_{j}\right\}$. Using Lemma 7 repeatedly, we can then say that $\pi^{d}=\pi_{1}^{d}+\ldots+\pi_{n}^{d}$ for valid configurations $\pi_{i}^{d}$ of $D_{i}$. Since $\pi^{r} \in \Phi\left(\pi^{d}\right)$, by definition of conformance mappings, $\pi^{r}$ must be a valid configuration of $R_{1}\|\ldots\| R_{n}$; hence $\pi^{r}=\pi_{1}^{r}+\ldots+\pi_{n}^{r}$ (Lemma 7), such that $\pi_{i}^{d} \in \Phi\left(\pi_{i}^{r}\right)$, for valid configurations $\pi_{i}^{r}$ of $R_{i} ; \pi^{r}$ is valid means $\pi^{r} \models \bigwedge_{S \subseteq\{1,2, \ldots, n\}} \rho_{S}^{r}$.

Given the afore-obtained result, we show that the QBF $\Psi$ is satisfiable. The LHS of the QBF $\Psi$ is the formula $\varphi_{1,2, \ldots, n}^{d}$, which is the conjunction $\rho_{S}^{d}$ for all subsets $S$ of $\{1,2, \ldots, n\}$. The forall quantifier outside would thus evaluate all configurations of $D_{1}\|\ldots\| D_{n}$ that satisfy $\varphi_{1,2, \ldots, n}^{d}$, i.e., they satisfy $\bigwedge_{S \subseteq\{1,2, \ldots, n\}} \rho_{S}^{d}$ : hence, all valid configurations of $D_{1}\|\ldots\| D_{n}$.
For the QBF to hold good, for all valid configurations of $D_{1}\|\ldots\| D_{n}$ that have been evaluated on the LHS, we must find some configuration of $R_{1}\|\ldots\| R_{n}$ that satisfies $\Phi_{1} \wedge \ldots \wedge \Phi_{n} \wedge \varphi_{1,2, \ldots, n}^{r}$ : (i) any configuration $\pi$ of $R_{1} \|$ ... $\| R_{n}$ that satisfies $\varphi_{1,2, \ldots, n}^{r}$ would be valid; (ii) further, if it has to satisfy $\Phi_{1} \wedge \ldots \wedge \Phi_{n}$, it must agree with $\pi_{i}^{r} \in$ $\Phi_{i}\left(\pi_{i}^{d}\right)$ over $\operatorname{Var}\left(R_{i}\right)$ for all $1 \leq i \leq n$. By Lemma 7 , this means that $\pi$ can be written as $\pi_{1}^{r}+\ldots+\pi_{n}^{r}$. Thus, for the QBF to hold, we must be able to find, for each valid configuration $\pi^{d}$ of $D_{1}\|\ldots\| D_{n}$, a valid configuration $\pi^{r}$ of $R_{1}\|\ldots\| R_{n}$ that can be written as $\pi_{1}^{r}+\ldots+\pi_{n}^{r}$, where $\pi_{i}^{r} \in \Phi_{i}\left(\pi_{i}^{d}\right)$ for each $i$. However, this is exactly what the mapping $\Phi$ that checks for conformance of $D_{1}\|\ldots\| D_{n}$ with $R_{1}\|\ldots\| R_{n}$ does. Since we assume that $\Phi$ exists, the QBF holds.

The converse can be shown in a similar way: that is, if the QBF $\Psi$ holds, then $D_{1}\|\ldots\| D_{n}$ will conform to $R_{1}\|\ldots\| R_{n}$.

\section{Tool and case studies}

We have implemented the feature and system level verification method in the tool SPLEnD (SPL Engine for Design variability verification) [13]. We give a brief overview of the tool here.

The architecture of SPLEnD is shown in figure 9. The tool SPLEnD, in its simplest form, takes one main input file, which contains a list of pairs of design (FSMd) and requirement(FSMr) for features. For each pair, there are three files associated: (i) an XML file representing FSMd, (ii) an XML file representing FSMr and (iii) a text file containing global predicates $\rho$. SPLEnD can perform conformance check at (i) feature level and (ii) SPL level or system level. To perform feature level verification, user can select a pair and click a Run button to do the conformance check. For the selected feature, SPLEnD encodes corresponding FSMd and FSMr into ProMeLa format [14]. This ProMeLa encoded file is input to SPIN. While running SPIN, SPLEnD captures all the conformance mappings between FSMd and FSMr. The tool SPLEnD performs the feature level verification of each pair using SPIN. This verification results in a suitable configuration mapping for every pair. For SPL level conformance check, users can also add global constraints $\rho$. The SPL level conformance

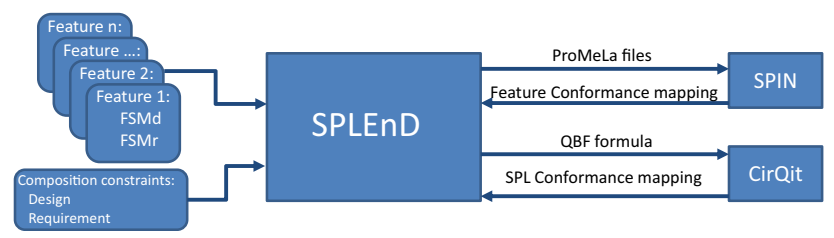

Figure 9. Architecture of SPLEnD. 
check is then performed by constructing the QBF formula from the configuration mapping information and using the CirQit QSAT Solver [15] for satisfiability checking.

\subsection{Creating or loading SPL model}

Figure 10 shows the main Graphical User Interface (GUI) for tool SPLEnD. Here, users are allowed to create or load SPL model with various files (mapping file, FSMd and FSMr for feature, constraints file). The figure shows that we have loaded ECPL SPL model and it displays the mapping for all features in SPL model. On the left side of screen it displays all the files in hierarchy. Bottom part of the screen displays the results and $\operatorname{logs}$ for conformance check.

\subsection{Feature level conformance check}

Figure 11 shows the screen for conformance check at feature level. This check is done for DepositMoney feature for Banking Software Product Line (BSPL). The BSPL is discussed in upcoming part of this section. The output window in bottom part of screen shows the list of configurations (design and requirement), list of conformance mapping and execution time. There is no design configuration that is not mapped with any of the requirement configurations. In figure 12, the bottom part of screen (output window) shows two invalid configurations for feature WithdrawMoney from BSPL. Also the screen shot shows FSMd projects for invalid configurations.

\subsection{System level conformance check}

Figure 13 shows the output for system level conformance check done on ECPL. The output window shows that the system level conformance check failed and displays the invalid configuration at SPL level.

\subsection{Model checking of single variants}

SPLEnD provides a few extra features, like allowing users to use model checking with Linear Temporal Logic (LTL) [16] formula on a single variant of SPL model. SPLEnD can generate the SPIN model of the design of the entire SPL for a single variant and check for a given LTL property on this particular variant. This feature is helpful in case of SPL level non-conformance to analyse the behaviour of the non-conforming configuration returned by the second check. To generate a SPIN model for design variant, user should (i)load SPL model, (ii)conduct feature level conformance for each feature in SPL model and (iii)check SPL level conformance. After this, SPLEnD displays the screen shown in figure 14, where it loads all features with their variables and drop-down box with the values of those variables. On changing the value of those variables, at the top it shows the possible number of design configurations for that variant. At the bottom text box, users can enter the LTL property, which can be verified against a single model generated from the selected configuration variables. This LTL property verification is done by background model checker SPIN.

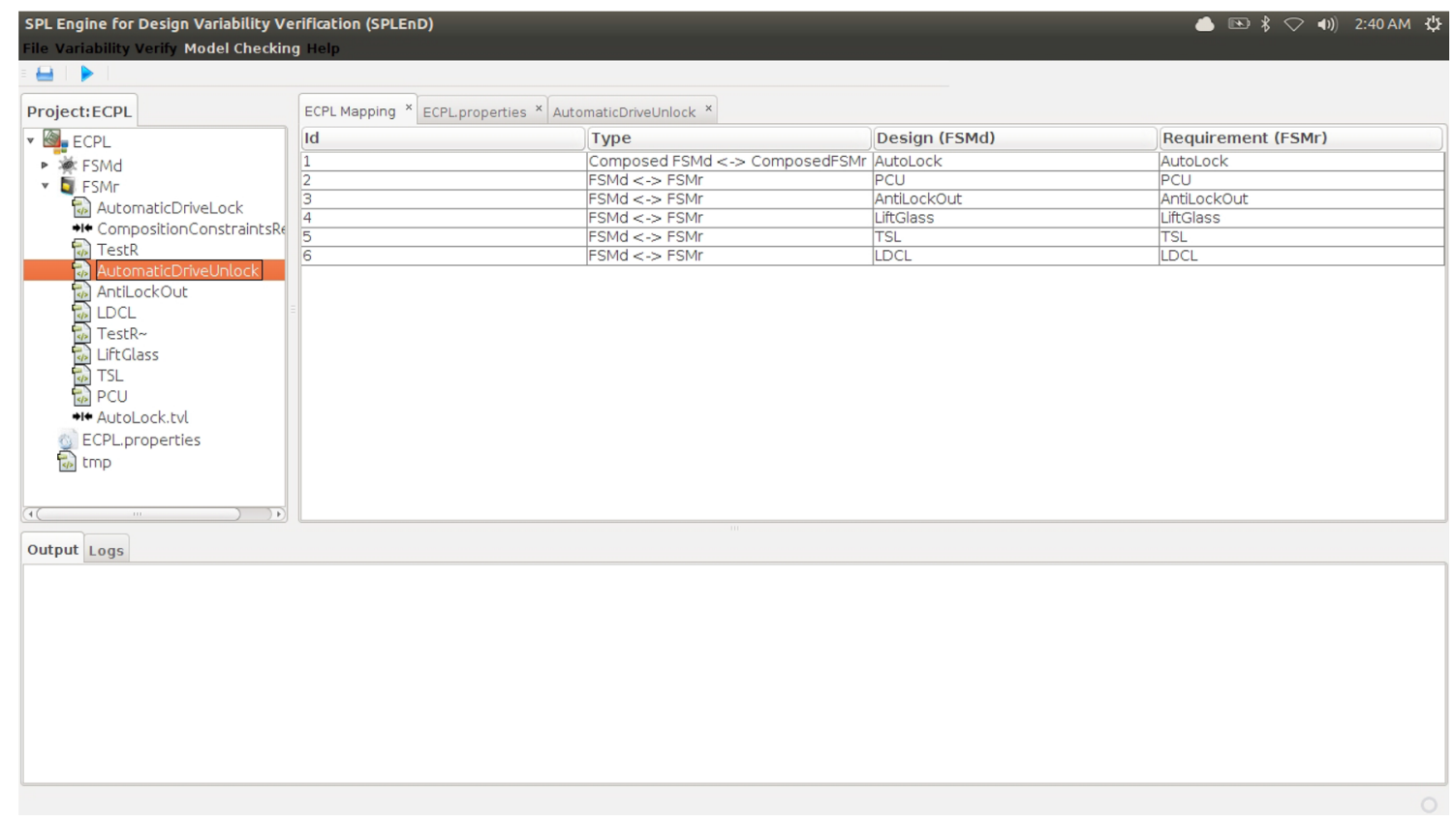

Figure 10. Loading SPL project screen in SPLEnD. 


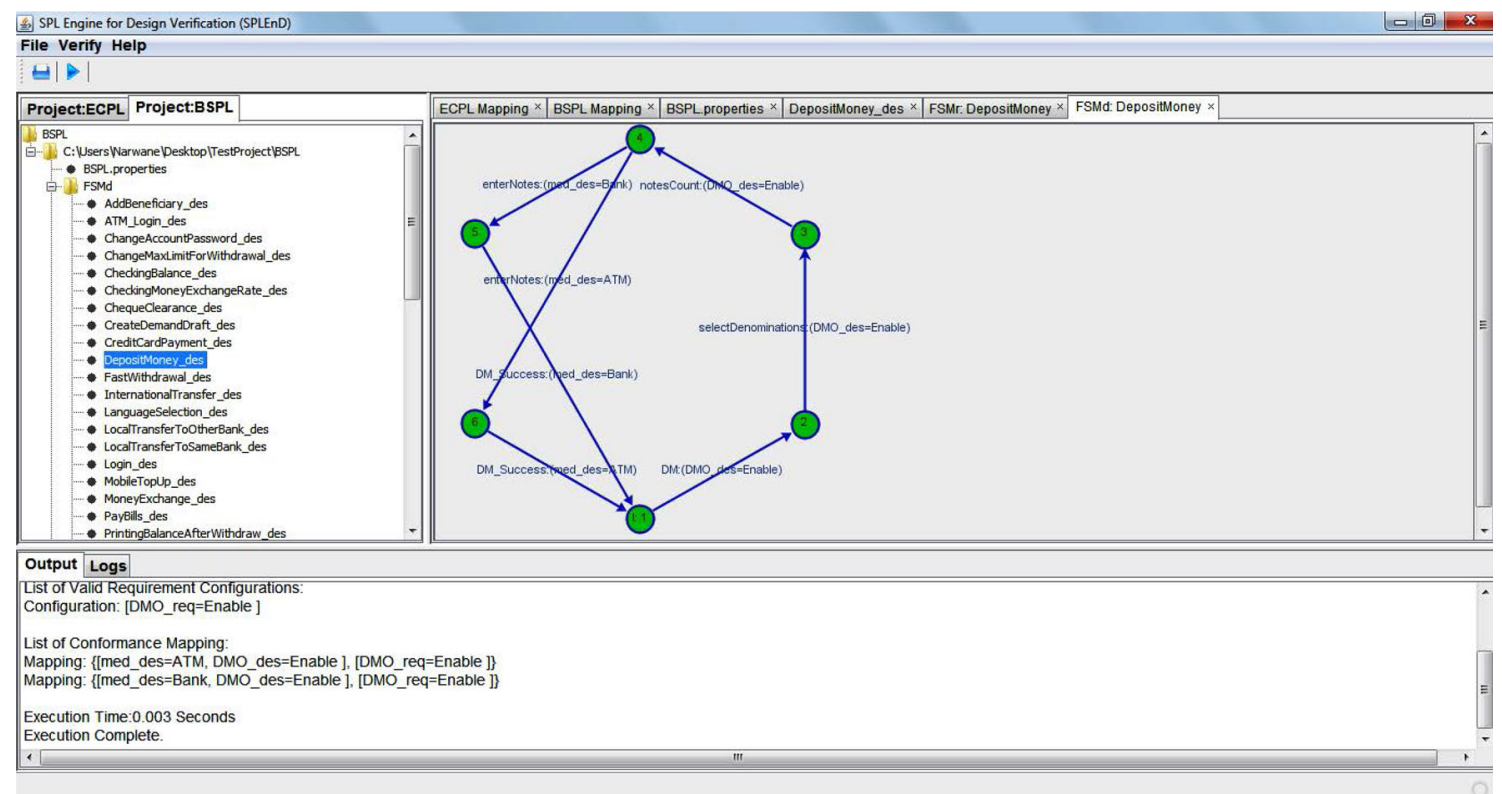

Figure 11. Screen showing feature level conformance check for valid case in SPLEnD.

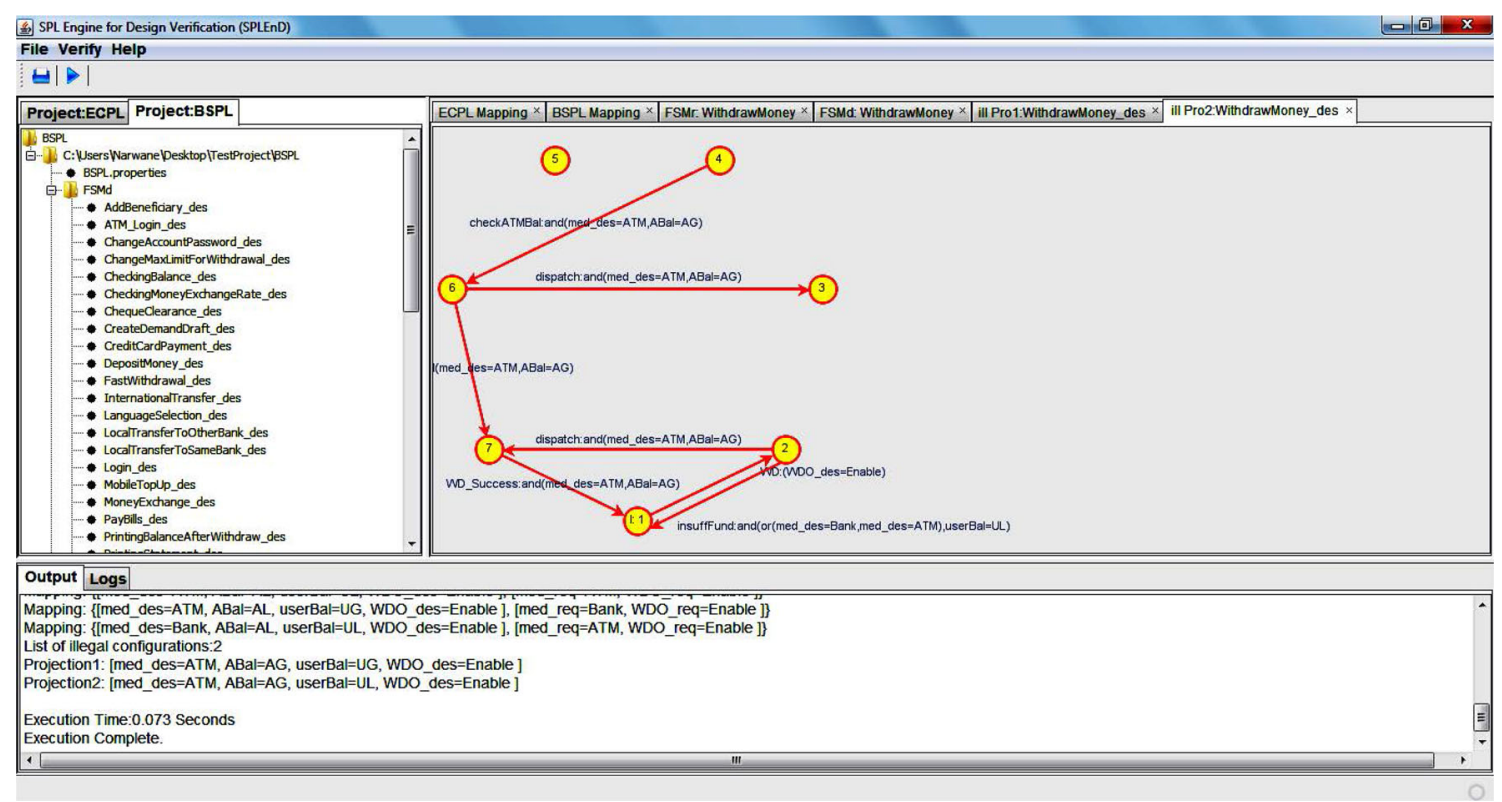

Figure 12. Screen showing feature level conformance check for invalid case in SPLEnD.

\subsection{Converting predicate}

One can give to SPLEnD a predicate defined using the vocabulary from the requirement level and SPLEnD translates it to the equivalent predicate at the design level using the individual conformance mapping. The purpose of this extra feature is to allow expressing properties (for model checking) at the requirement level where the variability is expressed naturally and get the automatic conversion to the design level vocabulary in order to check this property on the design model. Figure 15 shows the window that takes input as verification property at requirement level and allows converting it to design level.

In our experimentation with case studies, we found that each feature may be described by $n \geq 1$ requirement state machines and $m \geq 1$ design state machines, where $n$ and $m$ need not be the same. For example, in the ECPL case study, the AutoLock feature is composed of the features AutomaticDriveLock and AutomaticDriveUnlock and both have a variation point referring to the transmission of the car (automatic or manual). The group level composition 


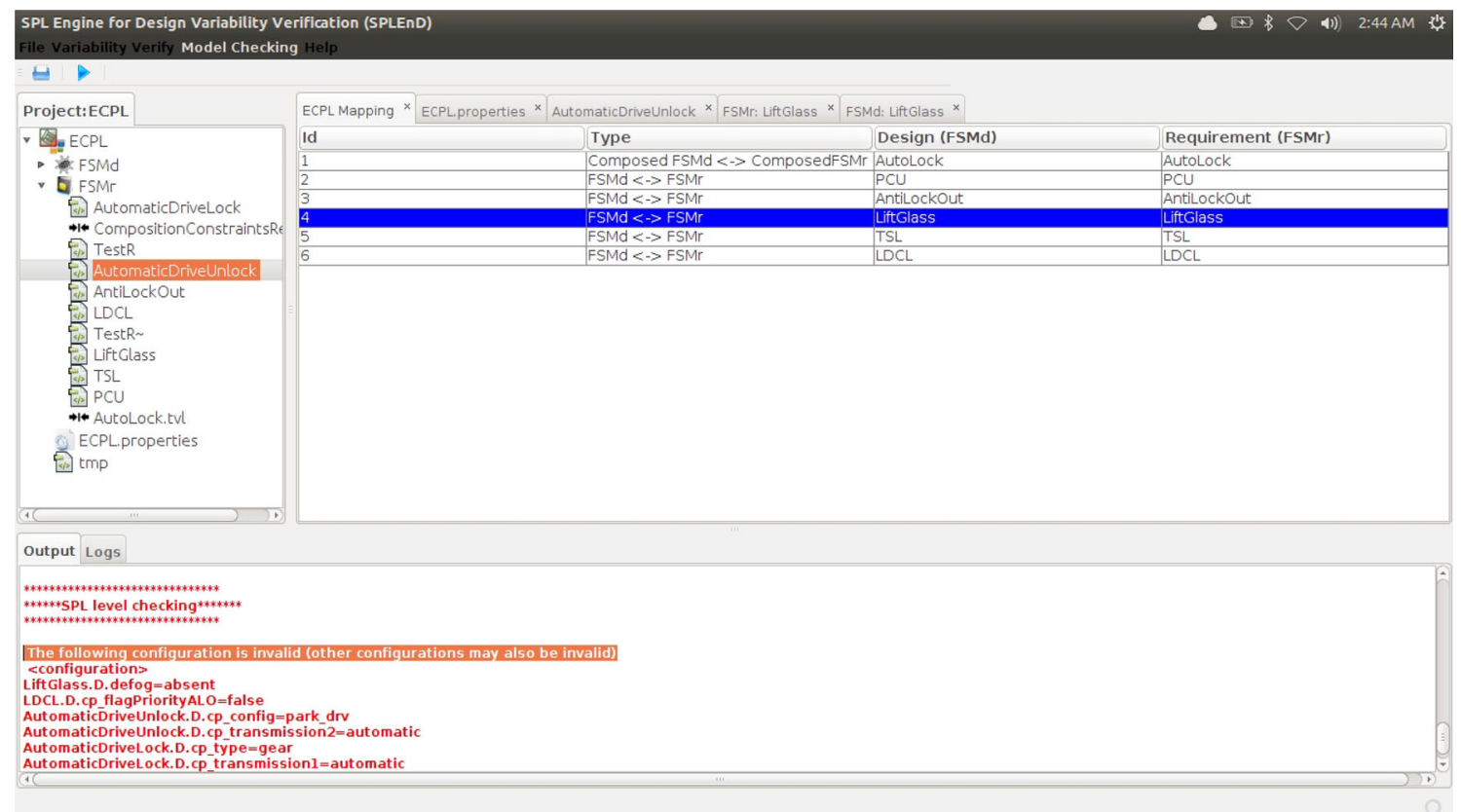

Figure 13. Screen showing system level conformance check for invalid case in SPLEnD.

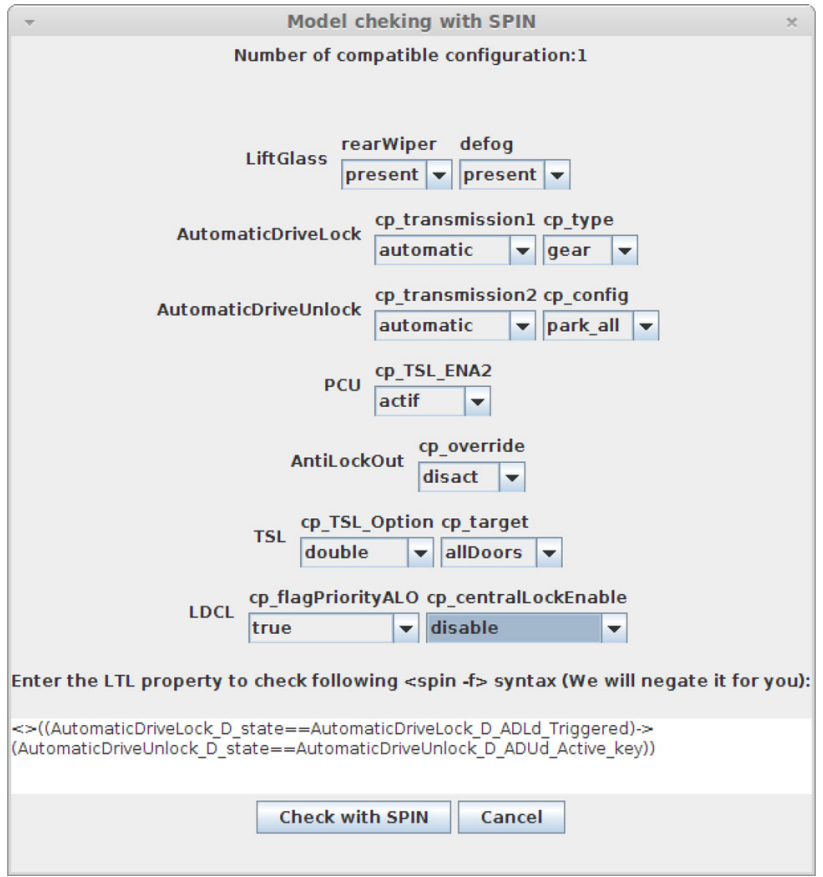

Figure 14. Snapshot of SPLEnD: select a variant, enter an LTL property and run SPIN.

constraint specifies that the transmission must have the same value in both features.

In order to take care of this case, we have implemented a pre-processing step, which composes each collection of requirements (and design) state machines into a single requirement (design) state machine before performing the

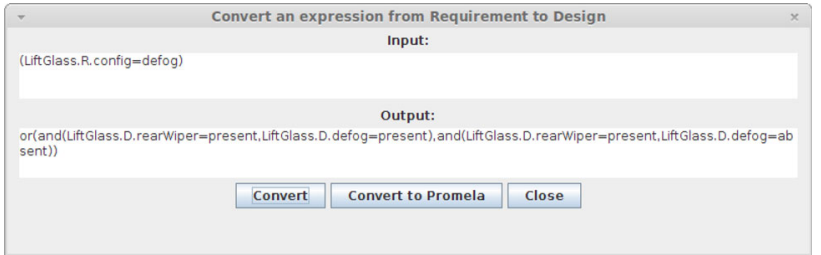

Figure 15. Snapshot of SPLEnD: converting a predicate from requirement to design.

\begin{tabular}{|c|c|c|}
\hline Features & $\begin{array}{c}\text { No. of Design } \\
\text { Configurations }\end{array}$ & $\begin{array}{c}\text { SPIN Exe. } \\
\text { Time (Sec) }\end{array}$ \\
\hline$P L \& L D C L$ & 8 & 0.93 \\
\hline$P C U$ & 3 & 0.05 \\
\hline AutomaticDriveLock & 2 & 0.11 \\
\hline AutomaticDriveUnlock & 4 & 0.24 \\
\hline AntiLockOut & 3 & 0.01 \\
\hline TSL & 8 & 0.67 \\
\hline LiftGlass & 3 & 0.07 \\
\hline
\end{tabular}

Figure 16. Execution time of SPLEnD on Algorithm 1 for ECPL.

feature level verification followed by the system level verification. Hence, in general, each pair input to SPLEnD may contain one or more requirement state machines and one or more design state machines.

We evaluated SPLEnD on a few case studies using this version of the implementation, the results of which are summarized in the rest of this section. Experiments have been performed on a $2.24 \mathrm{GHz}$ i5 processor machine with 6-GB RAM. 


\subsection{Experimental results for ECPL}

ECPL has 8 features; each feature was modelled with an (FSMd, FSMr) pair with less than 10 states and 3-4 variables. The time taken for each feature can be seen in figure 16. The second step involving synthesizing and solving a QBF formula took $0.04 \mathrm{~s}$ to complete.

\section{$5.7 B S P L$}

BSPL, taken from [17], is an SPL of a banking software that manages ATM and online banking transactions. Of the 176 features, 100 behavioural features were considered for our experimentation. Figure 17 presents the feature diagram of the BSPL.

5.7a The feature Withdraw Money: Figure 18 is the FSMr for the feature Withdraw Money. The events are \{enterAmount, insuffUserBal, insuffATMBal, checkATMBal, Disburse,Dispatch,WD $\}$. The Var for figure 18 is $\{W D O=$ $\{$ Enable,Disable $\}$, med $=\{$ Bank, ATM, Online $\}\}$ and global predicate $\rho$ is $\{(W D O=$ Enable $\wedge \neg($ med $=$ Online $)\}$.

Figure 19 is the FSMd for the feature Withdraw Money. This FSMd shares all the events of the FSMr. The Var for figure 19 is $\quad\{W D O=\{$ Enable, Disable $\}, \quad$ med $=$ $\{$ Bank, ATM, Online $\}, \quad p 1=\quad\{$ true, false $\}, \quad p 2=$ $\{$ true, false $\}, p 3=\{$ true, false $\}, p 4=\{$ true, false $\}\}$. The global predicate $\rho$ is $\{(W D O=$ Enable $\wedge \neg$ $($ med $=$ Online $)\}$. The Boolean variable $p 1$ represents predicate $(a m t \leq u s e r B a l), \quad p 2$ represents predicate $($ amt $>$ userBal $), p 3$ represents predicate $(a m t \leq A T M B a l)$ and $p 4$ represents predicate $(a m t>A T M B a l)$. We assume that the value for variables $p 1-p 4$ is true depending on whether a predicate is satisfied or not.

After running SPLEnD on feature Withdraw Money, we get the illegal configuration in design as $\pi=\{W D O=$ Enable, med $=$ ATM,$p 1=$ True, $p 2=$ False, $p 3=$ True,

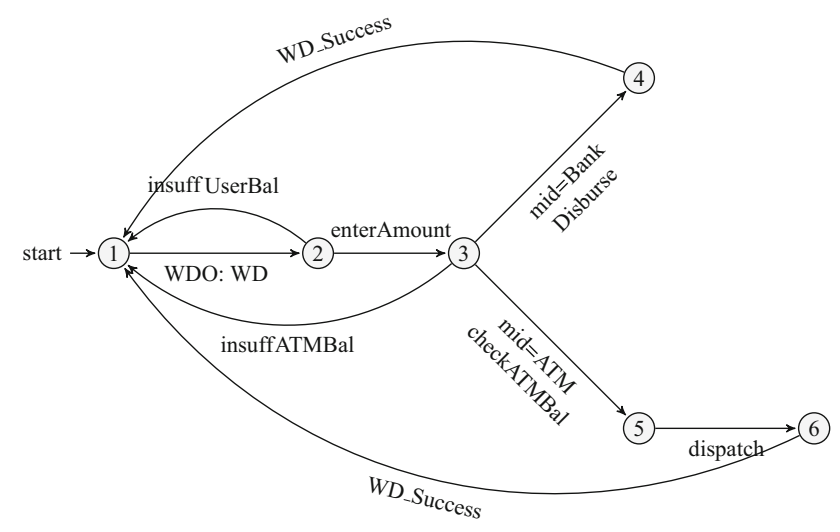

Figure 18. FSMr for the feature WithdrawMoney.

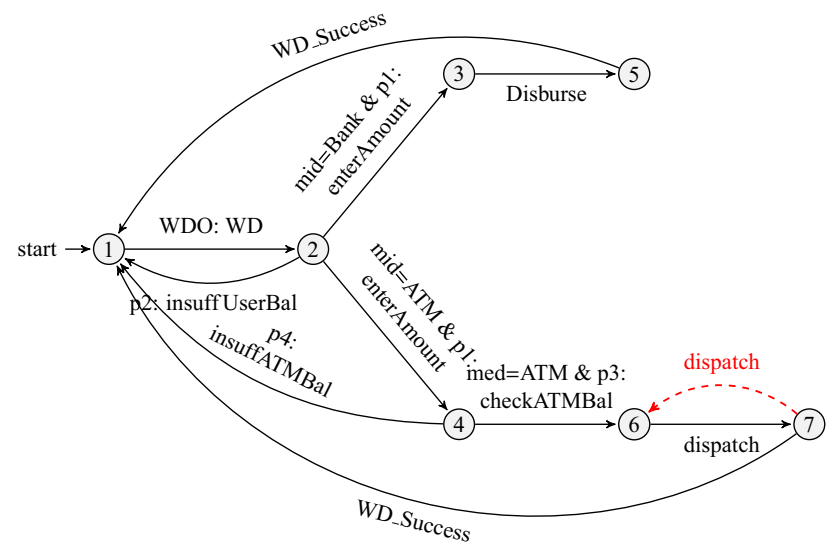

Figure 19. FSMd for the feature WithdrawMoney.

$p 4=$ False $\}$. When we project this configuration on the FSMd, we get an FSM as shown in figure 20.

It can be seen that the language of this FSM cannot be contained in any of the variants of the FSMr in figure 18.

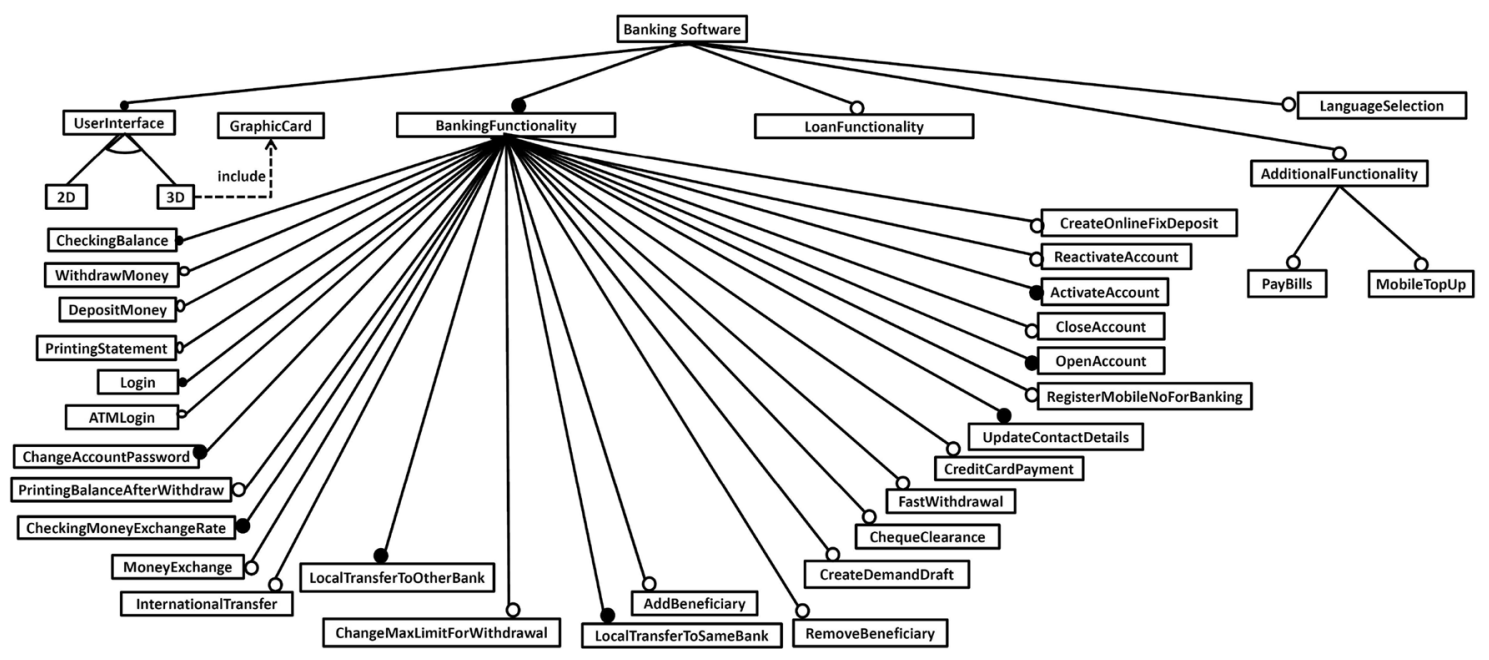

Figure 17. The feature diagram of the BSPL. 


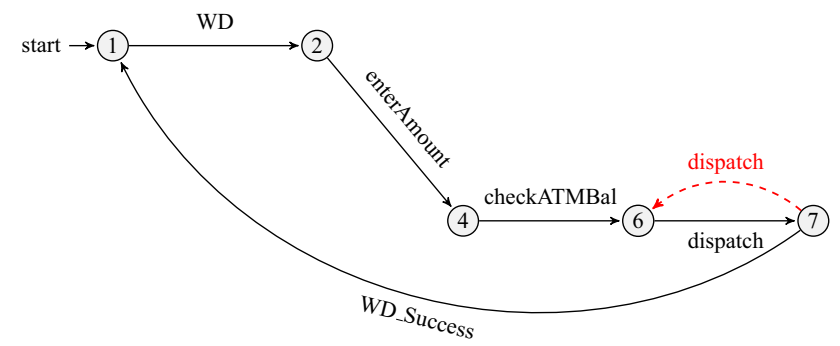

Figure 20. The behaviour of the invalid configuration of Withdraw Money.

Hence, we removed the transition in red from figure 19, to obtain conformance. With this modified FSMd, we carried out the rest of the study.

5.7b Overall experimental results for BSPL: The FSMv modelling the requirements and the designs of the features contain at most 8 states and have $2-8$ variables. Similar to ECPL, we ran Algorithm 1 on all the 100 features of BSPL. Figure 21 presents the number of design configurations and execution time of Algorithm 1 for each feature. The total time taken for performing the feature level conformance check over all the features is $5.47 \mathrm{~s}$; the individual times are given in figure 21. For the SPL level verification, SPLEnD synthesized the QBF formula having 960 variables. The checking of the QBF formula took $0.32 \mathrm{~s}$.

\subsection{Scalability check}

Encouraged by the result from BSPL, we checked the scalability of our approach by randomly generating SPL models with 5000-25,000 features. The FSMd, FSMr in each case had 3-8 states and 2 variables each. In this model, guards and global predicate $\rho$ are randomly generated over variables. The conformance check at the feature level was completed in less than $0.25 \mathrm{~s}$ in each case; the synthesized QBF formula had 10,000-50,000 variables. For example, in case of an SPL model with 5000 features and 2 variables for each feature, the QBF formula at SPL level conformance check generates 10000 variables. Figure 22 shows the time taken in seconds in each case by SPLEnD. The Variables row gives the number of variables in the design and requirement.

As the table shows, thanks to the QBF formulation, system level verification of large SPLs is possible.

\section{Related work}

Feature-based analysis: [18] explores feature-aware verification to automatically detect feature interactions in a software product line. A language was developed to specify individual features in separate and composable units; based on these feature-local specifications, feature interactions were detected in a product line by either (i) generating all the products and checking them one by one or (ii) generating one product that contains all the features. The email product line with 10 features and 40 products, with 27 feature interactions, was checked. [19] presents an approach oriented on programming language, which presents a core calculus for feature composition. The features may contain various kinds of software artefacts, like source code in various languages, models and documents. The composition is done uniformly across features with different artefacts in a type-safe way. [20] view features as state machines, and CTL model checking is used to verify properties of individual features. Compositional verification of features is done by checking the consistency of interface labels assigned by the CTL model checking algorithm at the feature level.

Behavioural conformance: [21] proposes the use of modal transition systems (MTS) over labelled transition systems for modelling and analysis of product line architectural behaviour. MTS can model optional and required behaviour via may and must transitions. A conformance algorithm for MTS is then presented: a fixed point algorithm that computes Cartesian product of states, and eliminates pairs that are invalid according to the relation.

The FTS $^{+}$proposed by [6] has some similarities with FSMv, but has a motivational difference. The aim of FTS ${ }^{+}$ is to model the entire SPL and hence there is a single global machine with a single global vocabulary for expressing variabilities; the variability information represents the presence/absence of features in the SPL. In contrast, our approach is based upon a different view of SPL: a feature with variability is an increment in functionality and an SPL is a collection of features. We use a single FSMv to model a feature and a whole SPL is modelled as a parallel composition of FSMv machines. The difference in viewpoint has another consequence: FTS $^{+}$models, since they model the entire SPL, tend to be large and hence have high analysis complexity; some abstraction techniques are hence used [22]. However, each FSMv models a fraction of functionality and hence can be analysed easily. [23] uses MTS for modelling product behaviour and uses the logic MHML for model checking. The approaches in [23] as well as [6] use transition systems for expressing system behaviour; feature variability constraints are expressed using feature diagrams in [6], while in [23], MHML is used to do this. [6] needs an extra component, a logic for checking properties, while in the case of [23], the MTS+MHML framework is sufficient.

Compositional verification: [24] proposes compositional verification for hierarchical SPLs. Here, Simple Hierarchical Variability Models (SHVMs) are used to specify the variability of product artefacts. However, in an SHVM, the number of derivable products is restricted by the fact that there is no means of defining constraints between variation points. [25] uses Event-B composition techniques for feature-based product line development. A 


\begin{tabular}{|c|c|c|c|}
\hline Features(Design Variants) & $\begin{array}{l}\text { Time } \\
(\mathrm{ms})\end{array}$ & Features(Design Variants) & $\begin{array}{l}\text { Time } \\
(\mathrm{ms})\end{array}$ \\
\hline OpenAccount(5) & 4 & ActivateAccount(5) & 3 \\
\hline ReactivateAccount(5) & 3 & CloseAccount (5) & 2 \\
\hline FreezeAccount $(5)$ & 2 & AddBeneficiary(5) & 2 \\
\hline ATMLogin(5) & 1 & BankEmployeeLogin(5) & 1 \\
\hline ChangeATMPin(5) & 3 & ChangeMaxLimit...(5) & 2 \\
\hline ChangePassword(5) & 2 & CheckingBalance(5) & 1 \\
\hline CheckingMoneyExchangeRate(5) & 3 & CreateDemandDraft(5) & 3 \\
\hline CreateDemandDraftOnline(5) & 3 & CreditCardPayment(5) & 2 \\
\hline CurrencyExchange(5) & 4 & DepositMoney(5) & 2 \\
\hline FastWithdrawal(13) & 25 & InterBankTransfer(4) & 4 \\
\hline InternationalTransfer(5) & 4 & IntraBankTransfer(5) & 3 \\
\hline LanguageSelection(5) & 1 & MobileTopUp(5) & 3 \\
\hline MoneyExchange(5) & 4 & OnlineCustomerLogin(5) & 1 \\
\hline PayBills(5) & 3 & PrintingBalanceAfterWithdraw(5) & 3 \\
\hline PrintingBankStatement(5) & 3 & RemoveBeneficiary(5) & 3 \\
\hline UpdateContactDetails(5) & 2 & UserInterface(5) & 2 \\
\hline WithdrawMoney(13) & 27 & ChequeClearance(5) & 3 \\
\hline RegisterMobileNoForBanking(5) & 2 & CreateOnlineFixDeposit(5) & 2 \\
\hline ApplyForLoanOnline(5) & 4 & EMICalculator(4) & 3 \\
\hline LoanEligibilityChecker(5) & 2 & UploadLoanDocuments(5) & 2 \\
\hline VerifyLoanDocuments(5) & 2 & VerifyLoanEligibility(5) & 2 \\
\hline SanctionLoan(5) & 1 & DisburseLoanAmount(5) & 1 \\
\hline LoanRepaymentEMI(5) & 2 & LoanRepaymentForecloser(5) & 2 \\
\hline AddAccountHolder(5) & 1 & DeleteAccountHolder(5) & 1 \\
\hline IssueChequeBook(5) & 2 & CreateStandingInstruction(5) & 2 \\
\hline DeleteStandingInstruction(5) & 2 & CreateECSTransaction(5) & 2 \\
\hline DeleteECSTransaction(5) & 2 & StopChequePayment(5) & 3 \\
\hline PayThroughRTGS(11) & 20 & PayThroughNEFT(11) & 22 \\
\hline CreditThroughRTGS(5) & 3 & CreditThroughNEFT(5) & 3 \\
\hline ReconcilingCardsTransactions(9) & 15 & ReconcilingATMTransactions(9) & 12 \\
\hline ScanSignatureToAccount(5) & 2 & MapDebitCardToAccount(5) & 2 \\
\hline MapChequeBookToAccount(5) & 2 & AutoATMPinForDebitCard(5) & 2 \\
\hline CloseFixedDeposit(5) & 1 & PreclosureFixedDeposit(5) & 1 \\
\hline AddCoBorrower(5) & 2 & AssetVerification(5) & 2 \\
\hline BlockDebitCard(5) & 2 & CancelDemandDraft(5) & 1 \\
\hline CreatePayOrder(5) & 2 & CancelPayOrder(5) & 1 \\
\hline LoadCashInATM(5) & 2 & DebitAnnualChargesOnDebCard(5) & 3 \\
\hline ApplyForCreditCardOnline(5) & 1 & CheckCreditCardStatus(5) & 2 \\
\hline EligibilityChecker(5) & 1 & UploadDocuments(5) & 2 \\
\hline VerifyDocuments(5) & 2 & DispatchCreditCard(5) & 3 \\
\hline BlockCreditCard(5) & 1 & GeneratePinForCreditCard(5) & 2 \\
\hline IssueNewCreditCard(5) & 2 & RegisterCardOnline(5) & 2 \\
\hline GeneratePhoneBankingPin(5) & 3 & CreditRewardPoints(5) & 2 \\
\hline RedemRewardPoints(5) & 3 & ConvertTransactionToEMI(5) & 4 \\
\hline PayCreditCardBill(5) & 4 & PayUtilityBills(5) & 4 \\
\hline ViewAccountSummary(5) & 2 & AddPayee(5) & 1 \\
\hline CreateStandingInst...(5) & 2 & ViewUnbilledTransaction(5) & 2 \\
\hline eStatement(5) & 2 & SendSMSAlerts(5) & 2 \\
\hline SendEmailAlerts(5) & 2 & GenerateOTP(5) & 1 \\
\hline RegisterForEstatement(5) & 1 & ApplyLateFees(5) & 2 \\
\hline
\end{tabular}

Figure 21. Execution time of SPLEnD on Algorithm 1 for BSPL. 


\begin{tabular}{|c|c|c|c|c|c|}
\hline Variables & $10^{4}$ & $2 \times 10^{4}$ & $3 \times 10^{4}$ & $4 \times 10^{4}$ & $5 \times 10^{4}$ \\
\hline Time $(\mathrm{sec})$ & 4.47 & 25.77 & 65.67 & 119.49 & 196.69 \\
\hline
\end{tabular}

Figure 22. Execution time of QBF for scalability.

feature is considered as a basic modular unit in the Rodin tool, and two case studies have been evaluated.

SAT solving: [26] was the first to propose the use of propositional logic for expressing relationships between requirements in a product line model. Using this, a product line model can be represented as a logical expression; this can be instantiated by the selected requirements. Further, it can be checked if the selected set is valid or not. [27] explores the fundamental connection between feature diagrams, grammars and propositional logic formulas. This connection paved the way for the use of SAT solvers that provide automated support to debug feature models.

Other approaches and SPL tools: Many other behavioural models have also been proposed [28-31], which are usually coupled with a variability model such as OVM [3], the Czarnecki feature model [2] or Variation Point Model (VPM) [32] to attain a fair level of variability expressibility. Unlike all these approaches, FSMv captures the variability in an explicit way, which we find more intuitive. The VPM of Gomaa and Olimpiew [32] distinguishes between variability at the requirement and design levels but no design verification approach has been presented. In a recent paper, Jorges et al [33] present a constraint-based approach for variability modelling. Here, architectural as well as behavioural constraints are captured using temporal logics; synthesis algorithms are then used to compute solutions. Berg et al [34] propose a model for variability handling throughout the life cycle of the SPL. Metzger et al [35] and Riebisch and Brcina [36] provide a similar approach but they do not consider the behavioural aspect. In our proposed approach, we extract the relation between requirement and design level variability from a behavioural analysis. [37] presents a tool VMC, for the modelling and analysis of product lines. The product family is represented as an MTS, along with extra variability constraints, and all the valid products are automatically generated. The tool implements the algorithm presented in [23]. A demonstration of the main features of VMC can be seen in [38]. Krishnamurthi and Fisler [39] have developed an analysis based on three-valued model checking of automata defined using step-wise refinement. Later on, Liu et al [40] revisited Fisler's approach to provide a much more efficient method. Recently, Cordy et al [41] extended Fisler's approach to LTL formula. Lauenroth et al [42], as well as Classen et al [6] and Cordy et al [22], and Gruler et al [43] have developed model checking methods for SPL behaviour. These methods are based on the verification of LTL/ CTL/modal $\mu$ calculus formula.
All these verification methods assume a global view of variability and hence the representation of variability information is identical in both specification and the design. By contrast, in our work the specification and design involve variability information at different levels of abstraction and hence one needs mapping information between the two levels. Furthermore, our formalism allows incremental addition of functionality and variability and enables compositional verification.

\section{Conclusion}

This paper introduced a new notion called design variability verification for checking the variability information expressed differently at the requirement and design levels. A method extending the classical finite state machine verification is developed for design variability verification. The proposed method is compositional and involves a combination of model checking and QBF SAT solving. A tool SPLEnD has been implemented and experimented with modest sized examples with encouraging results.

The conformance relation used in the paper is based on the language refinement notion. We plan to develop more discriminative relations along the lines of failure trace containment and bi-simulation relations. We are also experimenting with the tool capabilities for large industrial size SPL.

\section{Appendix}

In this section, we try to explain input models to SPLEnD using User Interface feature from Banking Software Product Line (BSPL). Figure 23 is the FSMr for feature User Interface, which has $U I$ as an event with global predicate $\rho=\{\neg($ uip $=$ Disable $)\}$. There is only one Boolean variable, Var $=\{$ uip : $\{$ Enable, Disable $\}$.

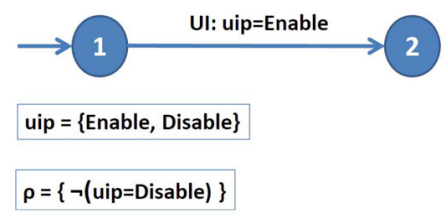

Figure 23. FSMr for feature: UserInterface.

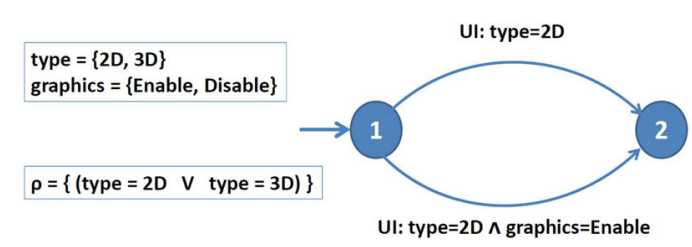

Figure 24. FSMd for feature: UserInterface. 
Figure 24 is the FSMd for feature User Interface. This FSMd shares the event $U I$ with the FSMr and has global predicate $\rho=\{($ type $=2 D \vee$ type $=3 D)\}$. There are two variables, Var $=\{$ type $:\{2 D, 3 D\}$, graphics : $\{$ Enable, Disable $\}$.

SPLEnD represents any FSMvs in XML format. The XML file corresponding to the FSMr in figure 23 is as follows:

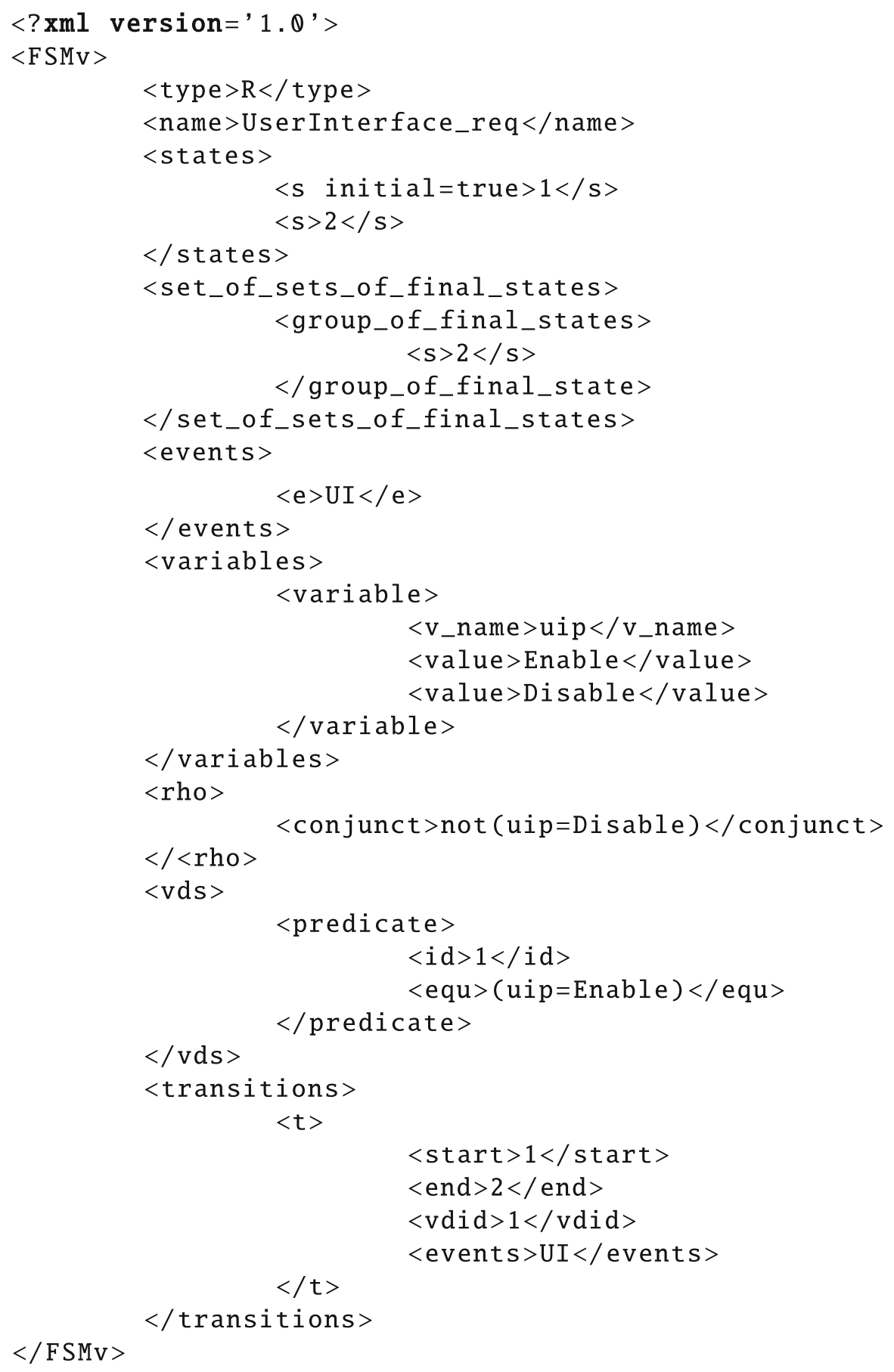


The XML file for the FSMd in figure 24 is as follows:

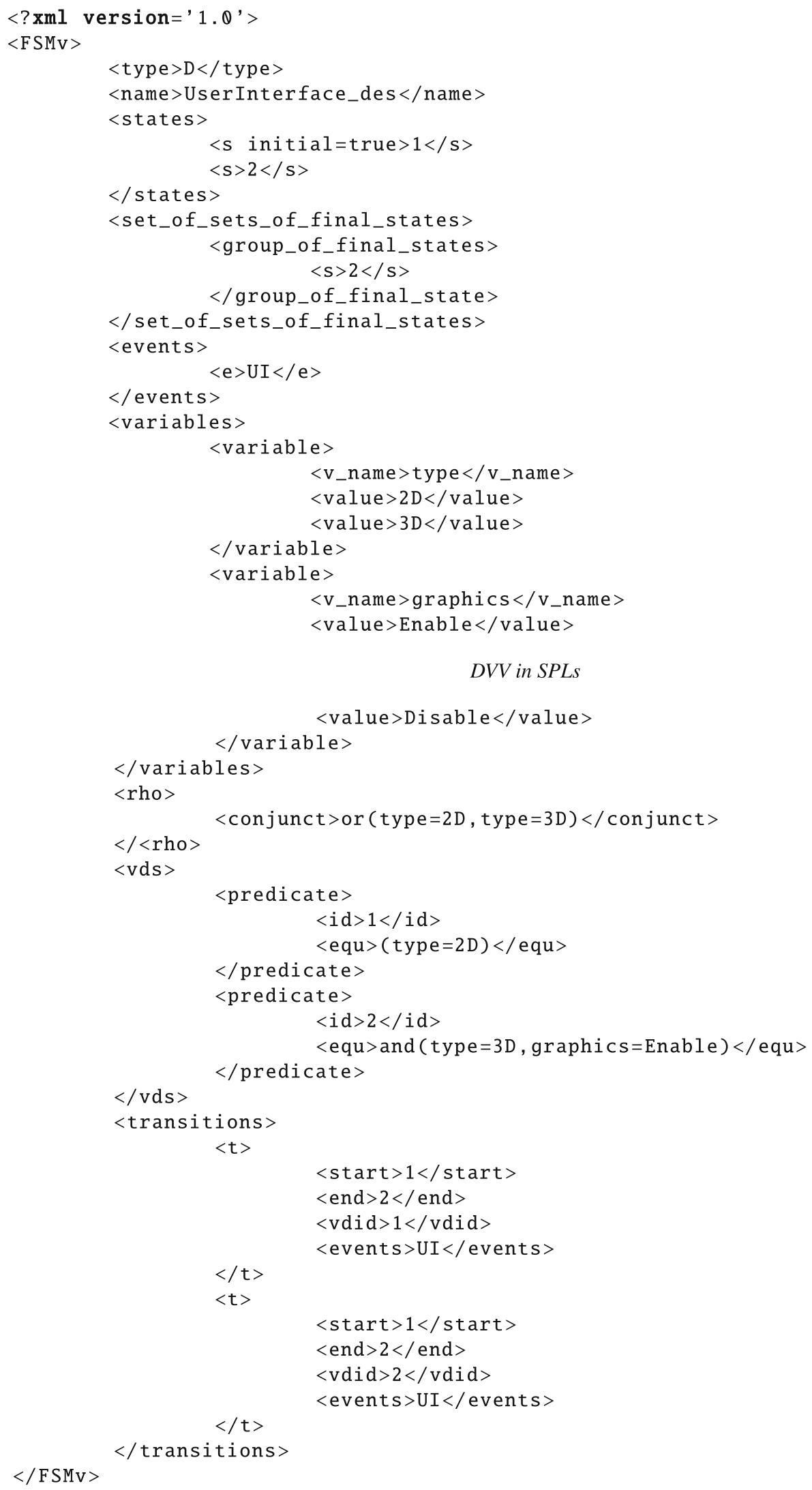




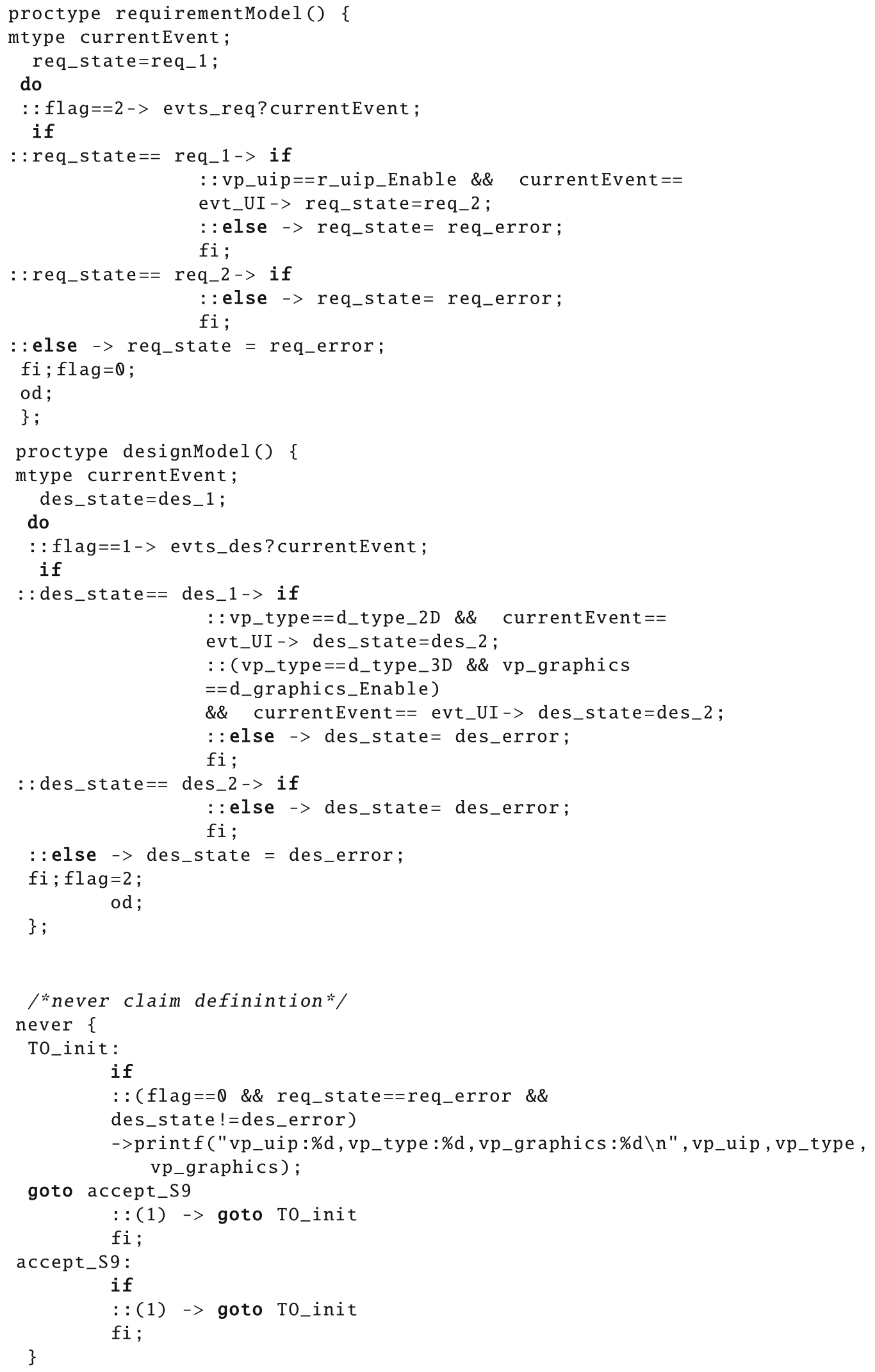




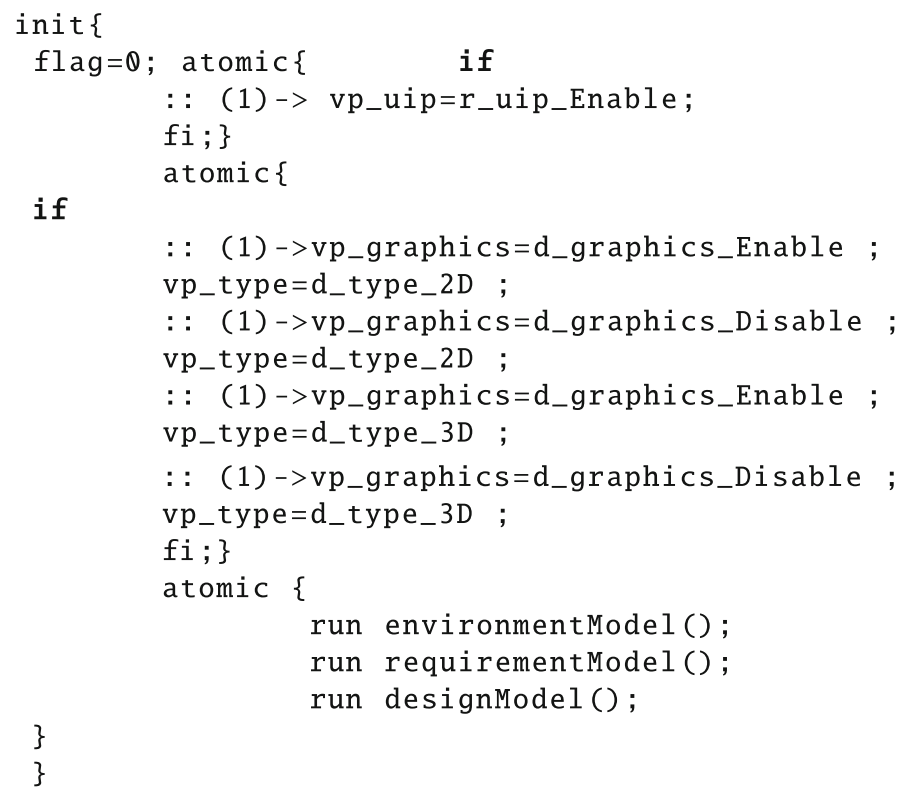

This ProMeLa model is given as an input to SPIN. Internally, SPIN generates pan.c (c program ) file corresponding to this ProMeLa model. SPIN executes pan.c to generate an executable file called as pan.exe; pan.exe is executed to generate the conformance mapping. SPLEnD collects conformance mapping results from SPIN. Similarly, $\Phi$ is constructed for all other features.

After construction of $\Phi$ for all features, SPLEnD created the QBF for the composite FSMd and FSMr. This QBF is converted to "qpro" format, which is an input format for CirQit QBF solver. CirQit verified this QBF, and returned the results to SPLEnD.

\section{References}

[1] Kang K C, Cohen S G, Hess J A, Novak W E and Spencer Peterson A 1990 Feature-oriented domain analysis (FODA) feasibility study. Technical Report CMU/SEI-90TR-21, Software Engineering Institute of Carnegie Mellon University, November

[2] Czarnecki K and Eisenecker U W 2000 Generative programming-methods, tools and applications. Addison-Wesley

[3] Metzger A and Pohl K 2007 Variability management in software product line engineering. In ICSE Companion, pp. 186-187

[4] Tischer C, Boss B, Müller A, Thums A, Acharya R and Schmid K 2012 Developing long-term stable product line architectures. In: Proceedings of the 16th International Software Product LineConference-SPLC '12, vol. 1, pp. 86-95. ACM, New York, NY, USA

[5] Cordy M, Schobbens PY, Heymans P and Legay A 2013 Beyond boolean product-line model checking: dealing with feature attributes and multi-features. In David Notkin, Betty H. C. Cheng, and Klaus Pohl, editors, ICSE, pp. 472-481. IEEE/ACM
[6] Classen A, Heymans P, Schobbens P Y and Legay A 2011 Symbolic model checking of software product lines. In: Proceedings of ICSE 2011, pp. 321-330

[7] ter Beek M H, Gnesi S and Mazzanti F 2013 VMC: a tool for the analysis of variability in software product lines. ERCIM News, 2013(93)

[8] Holzmann G J 2003 The SPIN model checker: primer and reference manual. Addison-Wesley Professional

[9] Goultiaeva A and Bacchus F 2010 Exploiting QBF duality on a circuit representation. In: Proceedings of $A A A I$

[10] Milner R 1982 A calculus of communicating systems. Springer, New York, Inc., Secaucus, NJ, USA

[11] Hoare C A R 1978 Communicating sequential processes. Commun. ACM 21(8): 666-677

[12] Vardi M Y and Wolper P 1986 An automata-theoretic approach to automatic program verification. In: Proceedings of LICS 1986, pp. 322-331

[13] Narwane G K, Millo J V, Ramesh S and Krishna S N 2016 SPLEnD website. https://www.cse.iitb.ac.in/ splend

[14] SPIN website. http://spinroot.com/spin/whatispin.html, 2010

[15] Goultiaeva A and Bacchus F 2010 CirQit website. http:// www.cs.utoronto.ca/ alexia/cirqit/,

[16] Huth M and Ryan M 2004 Logic in computer science: modelling and reasoning about systems. Cambridge University Press, New York, NY, USA

[17] Computer Systems Group/Generative Software Development Lab 2013 Softwareproduct lines online tools. University of Waterloo, Canada, October 2013

[18] Apel S, Speidel H, Wendler P, Rhein A and Beyer D 2011 Detection of feature interactions using feature-aware verification. In: Proceedings of ASE, pp. 372-375

[19] Apel S and Hutchins D 2010 A calculus for uniform feature composition. ACM Trans. Program. Lang. Syst. 32(5)

[20] Li H C, Krishnamurthi S and Fisler K 2002 Verifying crosscuttingfeatures as open systems. In: Proceedings of FSE, pp. 89-98 
[21] Fischbein D, Uchitel S and Braberman V 2006 A foundation forbehavioural conformance in software product line architectures. In: Proceedings of ROSATEA, pp. 39-48

[22] Cordy M, Classen A, Perrouin G, Schobbens P Y, Heymans P and Legay A 2012 Simulation-based abstractions for software product-line model checking. In: Proceedings of ICSE, pp. 672-682

[23] Asirelli P, terBeek M H, Gnesi S and Fantechi A 2011 Formal description of variability in product line families. In: Proceedings of SPLC, pp. 130-139

[24] Schaefer I, Gurov D and Soleimanifard S 2010 Compositional algorithmic verification of software product lines. In: Proceedings of FMCO, pp. 184-203

[25] Gondal A, Poppleton M and Butler M 2011 Composing event-B specifications-case study experience. In: Software Composition, pp. 100-115

[26] Mannion M 2002 Using first-order logic for product line model validation. In: Proceedings of SPLC, pp. 176-187

[27] Batory D S 2005 Feature models, grammars, and propositional formulas. In: J Henk Obbink and Klaus Pohl, editors, Proceedings of SPLC, volume 3714 of Lecture Notes in Computer Science, pp. 7-20. Springer

[28] Larsen K G, Nyman U and Wasowski A 2007 Modal i/o automata for interface and product line theories. In: Proceedings of the 16th European Conference on Programming, ESOP'07, pp. 64-79. Springer, Berlin, Heidelberg

[29] Raclet J B, Badouel E, Benveniste A, Caillaud B, Legay A and Passerone R 2009 Modal interfaces: unifying interface automata and modal specifications. In: Proceedings of EMSOFT, pp. 87-96

[30] Fantechi A and Gnesi S 2008 Formal modeling for product families engineering. In: SPLC'08, editor, Proceedings of SPLC'08, pp. 193-202. IEEE Computer Society

[31] Gruler A, Leucker M and Scheidemann K D 2008 Calculating and modeling common parts of software product lines. In: Proceedings of SPLC, pp. 203-212

[32] Gomaa H and Olimpiew E M 2008 Managing variability in reusable requirement models for software product lines. In: Proceedings of ICSR, pp. 182-185

[33] Jörges S, Lamprecht A L, Margaria T, Schaefer I and Steffen B 2012 A constraint-based variability modeling framework. Int. J. Softw. Tools Technol. Transf. 14(5): 511-530
[34] Berg K, Bishop J and Muthig D 2005 Tracing software product linevariability: from problem to solution space. In: Proceedings of the 2005 Annual Research Conference of the South African Instituteof Computer Scientists and Information Technologists on IT Researchin Developing Countries, SAICSIT '05, pp. 182-191. South African Institute for Computer Scientists andInformation Technologists, Republic of South Africa

[35] Metzger A, Heymans P, Pohl K, Schobbens P Y and Saval G 2007 Disambiguating the documentation of variability in software product lines: a separation of concerns, formalization and automated analysis. In: Proceedings of the $R E$ Conference, pp. 243-253

[36] Riebisch M and Brcina R 2008 Optimizing design for variability using traceability links. In: Proceedings of the ECBS Conference, pp. 235-244

[37] terBeek M H, Mazzanti F and Sulova A 2012 VMC: a tool for product variability analysis. In: Proceedings of the FM Symposium, pp. 450-454

[38] terBeek M H, Gnesi S and Mazzanti F 2012 Demonstration of a model checker for the analysis of product variability. In: Proceedings of SPLC, pp. 242-245

[39] Krishnamurthi S and Fisler K 2007 Foundations of incremental aspect model-checking. ACM Trans. Softw. Eng. Methodol. 16(2)

[40] Liu J, Basu S and Lutz R R 2011 Compositional model checking of software product lines using variation point obligations. Autom. Softw. Eng. 18(1): 39-76

[41] Cordy M, Schobbens P Y, Heymans P and Legay A 2012 Behavioural modelling and verification of real-time software product lines. In: Proceedings of SPLC, pp. 66-75

[42] Lauenroth K, Metzger A and Pohl K 2011 Quality assurance in the presence of variability. Technical report, SSE, Institut fur Informatik und Wirtschaftsinformatik, univertitat Duisburg Essen

[43] Gruler A, Leucker M and Scheidemann K 2008 Modeling and model checking software product lines. In: Proceedings of the 10th IFIP WG 6.1 International Conference on Formal Methods for Open Object-Based Distributed Systems, FMOODS '08, pp. 113-131. Springer, Berlin, Heidelberg 